\title{
Confluent Chains of DBT: Enlarged Shape Invariance and New Orthogonal Polynomials ${ }^{\star}$
}

Yves GRANDATI ${ }^{\dagger}$ and Christiane QUESNE $\ddagger$

† Equipe BioPhysStat, LCP A2MC, Université de Lorraine-Site de Metz, 1 bvd D.F. Arago, F-57070, Metz, France

E-mail: grandati@yahoo.fr

¥ Physique Nucléaire Théorique et Physique Mathématique, Université Libre de Bruxelles, Campus de la Plaine CP229, Boulevard du Triomphe, B-1050 Brussels, Belgium

E-mail: cquesne@ulb.ac.be

Received March 26, 2015, in final form July 15, 2015; Published online July 28, 2015

http://dx.doi.org/10.3842/SIGMA.2015.061

\begin{abstract}
We construct rational extensions of the Darboux-Pöschl-Teller and isotonic potentials via two-step confluent Darboux transformations. The former are strictly isospectral to the initial potential, whereas the latter are only quasi-isospectral. Both are associated to new families of orthogonal polynomials, which, in the first case, depend on a continuous parameter. We also prove that these extended potentials possess an enlarged shape invariance property.
\end{abstract}

Key words: quantum mechanics; supersymmetry; orthogonal polynomials

2010 Mathematics Subject Classification: 81Q05; 81Q60; 42C05

\section{Introduction}

Since the seminal paper of Gómez-Ullate, Kamran, and Milson [23], which introduced the concept of exceptional orthogonal polynomials (EOP), the discovery of their connection with translationally shape invariant quantum potentials (TSIP) by Quesne [41, 42], and the construction of infinite sets of such potentials by Odake and Sasaki [39], much progress has been made in the understanding of exactly solvable systems related to orthogonal polynomials (see [22] and references therein). The key tool to generate such systems is the Darboux or Darboux-Bäcklund transformation (DBT), which connects pairs of intertwined Hamiltonians. Starting from one primary TSIP, specific symmetries of this last select the quasi-polynomial formal eigenfunctions that can be used as seed functions to build chains of rationally extended potentials. The eigenstates of these extensions are then (up to a gauge factor) exceptional orthogonal polynomials, which, by using Crum formulas, can be expressed as Wronskians of classical orthogonal polynomials. The regularity properties of the chains, including degenerate chains (i.e., chains with repeated use of the same seed functions), are controlled by enlarged versions of the Krein-Adler theorem $[3,17,22,32,47]$. For some chains, the extended potentials share the same shape invariance properties as the primary potential [24, 25, 42, 43]. With other choices of seed functions, the resulting potentials possess an enlarged shape invariance property [26, 44, 45].

Until now, the chains of extensions were "rigid" in the sense that they were uniquely determined by the tuple of associated seed functions. Very recently, with B. Bagchi [4], we obtained new rational extensions of the Darboux-Pöschl-Teller potential (based on the so-called para-Jacobi polynomials [9]), which depend on a free parameter and can then be modulated

\footnotetext{
${ }^{\star}$ This paper is a contribution to the Special Issue on Exact Solvability and Symmetry Avatars in honour of Luc Vinet. The full collection is available at http://www.emis.de/journals/SIGMA/ESSA2014.html
} 
continuously. The eigenstates of these extended potentials are associated to new families of orthogonal polynomials that are, in a broad sense, exceptional para-Jacobi polynomials and which depend on a free continuous parameter.

In this paper, we consider the possibility of building new rational extensions of two confining TSIP, namely the trigonometric Darboux-Pöschl-Teller (TDPT) and isotonic potentials, via confluent chains of DBT, that is chains of DBT in which the spectral parameters of the different seed functions converge to the same value. It has to be noticed that it is precisely by using such confluent chains applied to the constant potential and considering the associated rational extensions that Adler and Moser built the Burchnall-Chaundy polynomials [8] in their seminal paper on the rational solutions of the KdV equation [2].

The possibility of considering two successive factorization energies tending towards a common real value in a chain of Darboux transformations was first considered in the framework of phaseequivalent potential construction [5], then extended to a class of potentials defined on the line [49]. These approaches generalized to arbitrary bound-state energies a procedure already known for the ground state and the first few excited states [13, 31]. An independent proposal was made wherein the terminology "confluent" algorithm was introduced and some partners of the free particle and the harmonic oscillator were exhibited [37]. The confluent algorithm was then studied in more general terms and applied to the free particle, one-soliton well, and harmonic oscillator [19]. It was also considered in a general construction of all possible first- and second-order partners of the TDPT potential [12]. The "hyperconfluent" third-order algorithm, wherein the three factorization energies converge to the same value, was analyzed [21]. Some Wronskian formulas applicable to the confluent case were also derived [7, 20, 48].

The present work differs from the previous ones devoted to confluent chains by the restriction to final potentials that are rational extensions of the initial ones. As a consequence of this condition, some parameters of the latter may have to be chosen integer.

After recalling in Section 2 the basic elements concerning the Darboux-Bäcklund transformations, we review in Section 3 the concept of confluent chains of DBT. We show in particular that the confluent chains of arbitrary order can be generated within the standard frame of (completed) DBT chains, giving rise to multiparameter dependent extensions.

In Section 4, applying two-step confluent chains of DBT for which the seed functions are eigenstates, we build regular rational extensions of the TDPT with appropriate parameters. The extended potentials depend on a continuous parameter and are strictly isospectral to the initial potential. The eigenstates form new families of orthogonal polynomials, which have a free parameter dependence. We exhibit particular examples and prove that the extended potentials present an enlarged shape invariance property, in which the parameter transformation acts in a nontrivial way on the supplementary parameter.

In Section 5, we make the same construction for the isotonic system. In contrast with the TDPT case, the regular rational extensions do not depend on any supplementary degree of freedom and we only have quasi-isospectrality between the extended potentials and the original one. We also furnish explicit examples of extensions and establish their enlarged shape invariance property. Section 6 contains some final comments.

\section{Darboux-Bäcklund transformations: basic elements}

We consider a one-dimensional Hamiltonian $\widehat{H}=-d^{2} / d x^{2}+V(x), x \in I \subset \mathbb{R}$, and the associated Schrödinger equation

$$
\psi_{\lambda}^{\prime \prime}(x)+\left(E_{\lambda}-V(x)\right) \psi_{\lambda}(x)=0,
$$

$\psi_{\lambda}(x)$ being a formal eigenfunction of $\widehat{H}$ for the eigenvalue $E_{\lambda}$. In the following, we suppose that, with Dirichlet boundary conditions on $I, \widehat{H}$ admits a discrete spectrum of energies and 


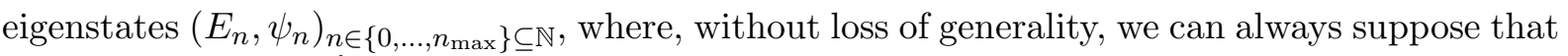
the ground level of $\widehat{H}$ is at zero $\left(E_{0}=0\right)$.

The Riccati-Schrödinger (RS) function $w_{\lambda}(x)=-\psi_{\lambda}^{\prime}(x) / \psi_{\lambda}(x)$ associated to $\psi_{\lambda}$ satisfies the corresponding Riccati-Schrödinger equation [28]

$$
-w_{\lambda}^{\prime}(x)+w_{\lambda}^{2}(x)=V(x)-E_{\lambda} .
$$

From any solution $\psi_{\nu}$ (or equivalently $w_{\nu}$ ), we can build a Darboux-Bäcklund transformation (DBT) $A\left(w_{\nu}\right)$ defined as $[10,11,15,16,28]$

$$
\begin{aligned}
& w_{\lambda}(x) \stackrel{A\left(w_{\nu}\right)}{\rightarrow} w_{\lambda}^{(\nu)}(x)=-w_{\nu}(x)+\left(E_{\lambda}-E_{\nu}\right) /\left(w_{\nu}(x)-w_{\lambda}(x)\right), \\
& \psi_{\lambda}(x) \stackrel{A\left(w_{\nu}\right)}{\rightarrow} \psi_{\lambda}^{(\nu)}(x)=\exp \left(-\int d x w_{\lambda}^{(\nu)}(x)\right) \sim \widehat{A}\left(w_{\nu}\right) \psi_{\lambda}(x), \quad \lambda \neq \nu,
\end{aligned}
$$

where $\widehat{A}\left(w_{\nu}\right)$ is a first-order differential operator given by

$$
\widehat{A}\left(w_{\nu}\right)=d / d x+w_{\nu}(x) .
$$

$\psi_{\lambda}^{(\nu)}$ and $w_{\lambda}^{(\nu)}$ are respectively solutions of the Schrödinger and RS equations with the same energy $E_{\lambda}$ as in equations (2.1) and (2.2), but with a modified potential

$$
V^{(\nu)}(x)=V(x)+2 w_{\nu}^{\prime}(x),
$$

that we call an extension of $V(x)$. For the associated Hamiltonian $\widehat{H}^{(\nu)}=-d^{2} / d x^{2}+V^{(\nu)}(x)$, we have the factorizations

$$
\widehat{H}^{(\nu)}=\widehat{A}\left(w_{\nu}\right) \widehat{A}^{+}\left(w_{\nu}\right)+E_{\nu}, \quad \widehat{H}=\widehat{A}^{+}\left(w_{\nu}\right) \widehat{A}\left(w_{\nu}\right)+E_{\nu},
$$

with

$$
\psi_{\lambda}(x) \sim \widehat{A}^{+}\left(w_{\nu}\right) \psi_{\lambda}^{(\nu)}(x)
$$

The function $\psi_{\lambda}^{(\nu)}$ in equation (2.3) can then be rewritten as the Darboux-Crum formula

$$
\psi_{\lambda}^{(\nu)}(x) \sim \frac{W\left(\psi_{\nu}, \psi_{\lambda} \mid x\right)}{\psi_{\nu}(x)}
$$

where $W\left(y_{1}, \ldots, y_{m} \mid x\right)$ denotes the Wronskian of the family of functions $y_{1}, \ldots, y_{m}$,

$$
W\left(y_{1}, \ldots, y_{m} \mid x\right)=\left|\begin{array}{ccc}
y_{1}(x) & \ldots & y_{m}(x) \\
\ldots & & \ldots \\
y_{1}^{(m-1)}(x) & \ldots & y_{m}^{(m-1)}(x)
\end{array}\right| .
$$

The eigenfunction $\psi_{\nu}$ is called the seed function of the DBT $A\left(w_{\nu}\right)$ and $V^{(\nu)}$ and $\psi_{\lambda}^{(\nu)}$ are the Darboux transforms of $V$ and $\psi_{\lambda}$, respectively.

Note that $A\left(w_{\nu}\right)$ annihilates $\psi_{\nu}$ and, consequently, equations (2.3) and (2.5) allow to obtain an eigenfunction of $V^{(\nu)}$ for the eigenvalue $E_{\lambda}$ only when $\lambda \neq \nu$. Nevertheless, we can readily verify that $1 / \psi_{\nu}(x)$ is such an eigenfunction. By extension, we then define the "image" by $A\left(w_{\nu}\right)$ of the seed eigenfunction $\psi_{\nu}$ itself as

$$
\psi_{\nu}^{(\nu)}(x) \sim \frac{1}{\psi_{\nu}(x)}
$$


At the formal level, the DBT can be straightforwardly iterated and a chain of $m$ DBT can be simply described by the following scheme

$$
\begin{gathered}
\psi_{\lambda} \stackrel{A\left(w_{\nu_{1}}\right)}{\longmapsto} \psi_{\lambda}^{\left(\nu_{1}\right)} \stackrel{A(\underbrace{\left(N_{1}\right)}_{\nu_{2}})}{\longmapsto} \psi_{\lambda}^{\left(N_{2}\right)} \ldots \stackrel{A\left(w_{\nu_{m}}^{\left(N_{m-1}\right)}\right)}{\longmapsto} \psi_{\lambda}^{\left(N_{m}\right)}, \\
V \stackrel{A\left(w_{\nu_{1}}\right)}{\longmapsto} V^{\left(\nu_{1}\right)} \stackrel{A\left(w_{\nu_{2}}^{\left(N_{1}\right)}\right)}{\longmapsto} V^{\left(N_{2}\right)} \ldots \stackrel{A\left(w_{\nu_{m}}^{\left(N_{m}\right)}\right)}{\longmapsto} V^{\left(N_{m}\right)},
\end{gathered}
$$

where $N_{j}$ denotes the $j$-uple $\left(\nu_{1}, \ldots, \nu_{j}\right)$ (with $N_{1}=\nu_{1}$ ), which completely characterizes the chain. We denote by $\left(N_{m}, \nu_{m+1}, \ldots, \nu_{m+k}\right)$ the chain obtained by adding to the chain $N_{m}$ the DBT associated to the successive eigenfunctions $\psi_{\nu_{m+1}}^{\left(N_{m}\right)}, \ldots, \psi_{\nu_{m+k}}^{\left(N_{m+k-1}\right)}$.

$\psi_{\lambda}^{\left(N_{m}\right)}$ is an eigenfunction associated to the eigenvalue $E_{\lambda}$ of the potential (see equation (2.4))

$$
V^{\left(N_{m}\right)}(x)=V(x)+2 \sum_{j=1}^{m}\left(w_{\nu_{j}}^{\left(N_{j-1}\right)}(x)\right)^{\prime}
$$

and can be written as (cf. equations (2.3) and (2.5))

$$
\psi_{\lambda}^{\left(N_{m}\right)}(x)=\widehat{A}\left(w_{\nu_{m}}^{\left(N_{m-1}\right)}\right) \psi_{\lambda}^{\left(N_{m-1}\right)}(x)=\widehat{A}\left(w_{\nu_{m}}^{\left(N_{m-1}\right)}\right) \cdots \widehat{A}\left(w_{\nu_{1}}\right) \psi_{\lambda}(x) .
$$

A chain is non-degenerate if all the spectral indices $\nu_{i}$ of the chain $N_{m}$ are distinct and is degenerate if some of them are repeated in the chain. For non-degenerate chains, Crum has derived very useful formulas for the extended potentials and their eigenfunctions in terms of Wronskians of eigenfunctions of the initial potential [14].

Crum's formulas. When all the $\nu_{j}$ and $\lambda$ are distinct, we have

$$
\psi_{\lambda}^{\left(N_{m}\right)}(x)=\frac{W^{\left(N_{m}, \lambda\right)}(x)}{W^{\left(N_{m}\right)}(x)}
$$

and

$$
V^{\left(N_{m}\right)}(x)=V(x)-2\left(\log W^{\left(N_{m}\right)}(x)\right)^{\prime \prime},
$$

where $W^{\left(N_{m}\right)}(x)=W\left(\psi_{\nu_{1}}, \ldots, \psi_{\nu_{m}} \mid x\right)$.

\section{Confluent chains of DBT}

The single-confluent limit of a chain of DBT $N_{m}$ is obtained when all the spectral indices $\nu_{j}$ tend simultaneously to the same value $\nu_{j} \rightarrow \nu, \forall j \in\{1, \ldots, m\}$ (in the following, we consider only single-confluent chains and than omit the adjective "single").

\section{$3.1 \quad$ Two-step confluent chains}

We consider a chain of two DBT $N_{2}=\left(\nu_{1}, \nu_{2}\right)$, which, in the non-degenerate case $\nu_{1} \neq \nu_{2}$, gives (see equations (2.7), (2.8), (2.9), and (2.10))

$$
\begin{aligned}
& V^{\left(\nu_{1}, \nu_{2}\right)}(x)=V(x)-2\left(\log W^{\left(\nu_{1}, \nu_{2}\right)}(x)\right)^{\prime \prime}=V(x)-2\left[\left(E_{\nu_{2}}-E_{\nu_{1}}\right) /\left(w_{\nu_{2}}(x)-w_{\nu_{1}}(x)\right)\right]^{\prime}, \\
& \psi_{\nu_{2}}^{\left(\nu_{1}\right)}(x)=\left(w_{\nu_{1}}(x)-w_{\nu_{2}}(x)\right) \psi_{\nu_{2}}(x)=W\left(\psi_{\nu_{1}}, \psi_{\nu_{2}} \mid x\right) / \psi_{\nu_{1}}(x) .
\end{aligned}
$$

Note that in the degenerate case $\nu_{1}=\nu_{2}$, we have (see equation (2.6))

$$
\psi_{\nu_{1}}^{\left(\nu_{1}\right)}(x)=1 / \psi_{\nu_{1}}(x), \quad w_{\nu_{1}}^{\left(\nu_{1}\right)}(x)=-w_{\nu_{1}}(x),
$$


and by applying the $\operatorname{DBT} A\left(w_{\nu_{1}}^{\left(\nu_{1}\right)}\right)=A\left(-w_{\nu_{1}}\right)$ to $V^{\left(\nu_{1}\right)}$, we recover simply the initial potential $V$,

$$
V^{\left(\nu_{1}, \nu_{1}\right)}(x)=V^{\left(\nu_{1}\right)}(x)-2 w_{\nu_{1}}^{\prime}(x)=V(x) .
$$

The confluent case corresponds to the limit $\nu_{2} \rightarrow \nu_{1}$. As proven by Fernández et al. [7, 20], the confluent extended potential and its eigenstates admit the following integral representations

$$
\widetilde{V}^{\left(\nu_{1}, \nu_{1}\right)}(x)=V(x)-2\left(\log \left(\int_{x_{0}}^{x} d t \psi_{\nu_{1}}^{2}(t)-W_{0}\right)\right)^{\prime \prime}
$$

and

$$
\widetilde{\psi}_{k}^{\left(\nu_{1}, \nu_{1}\right)}(x)=\left(E_{\nu_{1}}-E_{k}\right) \psi_{k}(x)-\frac{\psi_{\nu_{1}}^{2}(x)}{\int_{x_{0}}^{x} d t \psi_{\nu_{1}}^{2}(t)-W_{0}} \psi_{k}^{\left(\nu_{1}\right)}(x) .
$$

Both depend on an arbitrary real parameter $W_{0}$ and for an adapted range of $W_{0}$ values, the extended potential is regular. In fact, the formula for the potential (3.2) already appears in many previous works, for instance in a 1986 paper of Luban and Pursey [33] and a few years later in [31]. The Matveev formulas [27, 34, 35] for the two-step case

$$
\begin{aligned}
& \widetilde{V}^{\left(\nu_{1}, \nu_{1}\right)}(x)=V(x)-2\left[\log W\left(\psi_{\nu_{1}},\left(\frac{\partial \psi_{\nu}(x)}{\partial E_{\nu}}\right)_{\nu=\nu_{1}} \mid x\right)\right]^{\prime \prime}, \\
& \widetilde{\psi}_{k}^{\left(\nu_{1}, \nu_{1}\right)}(x)=W\left(\psi_{\nu_{1}},\left(\frac{\partial \psi_{\nu}(x)}{\partial E_{\nu}}\right)_{\nu=\nu_{1}}, \psi_{k} \mid x\right) / W\left(\psi_{\nu_{1}},\left(\frac{\partial \psi_{\nu}(x)}{\partial E_{\nu}}\right)_{\nu=\nu_{1}} \mid x\right),
\end{aligned}
$$

which express the confluent extension and its eigenstates in terms of generalized Wronskians (which are in fact two-way, or double Wronskians [51]) can be viewed as associated to a particular choice of the $W_{0}$ constant. Indeed, if we consider the indexed family of RS functions $w_{\nu}(x)$ as satisfying a prescribed initial condition in $x_{0}$, we have, in the confluent limit, $w_{\nu_{2}}(x) \rightarrow w_{\nu_{1}}(x)$. It results from equation (3.1) that

$$
V^{\left(\nu_{1}, \nu_{1}\right)}(x)=V(x)-2\left(1 /\left(\frac{\partial w_{\nu}(x)}{\partial E_{\nu}}\right)_{\nu=\nu_{1}}\right)^{\prime} .
$$

But we can readily verify that

$$
1 /\left(\frac{\partial w_{\nu}(x)}{\partial E_{\nu}}\right)_{\nu=\nu_{1}}=\left[\log W\left(\psi_{\nu_{1}},\left(\frac{\partial \psi_{\nu}(x)}{\partial E_{\nu}}\right)_{\nu=\nu_{1}} \mid x\right)\right]^{\prime}
$$

and since in this case we also have [36]

$$
\left(\frac{\partial w_{\nu}(x)}{\partial E_{\nu}}\right)_{\nu=\nu_{1}}=\frac{1}{\psi_{\nu_{1}}^{2}(x)} \int_{x_{0}}^{x} d t \psi_{\nu_{1}}^{2}(t)
$$

we see that equation (3.4) corresponds to equations (3.2) and (3.3) with $W_{0}=0$.

It has to be noticed that the degenerate extension $V^{\left(\nu_{1}, \nu_{1}\right)}(x)$ can be recovered from the confluent one $\widetilde{V}^{\left(\nu_{1}, \nu_{1}\right)}(x)$ by taking the singular limit value $W_{0} \rightarrow \infty$.

Fernández et al. used these formulas to generate new second-order SUSY partners of the free particle, the Kepler-Coulomb, and the single-gap Lamé potentials [7, 20].

The preceding results can be in fact integrated within the standard DBT scheme simply using the DBT in its completed form (see equation (2.6)) as in [13,31]. Indeed, by applying the DBT $A\left(w_{\nu}\right)$, we generate first the one-step (possibly singular) extension

$$
V^{(\nu)}(x)=V(x)+2 w_{\nu}^{\prime}(x)=V(x)-2\left(\log \psi_{\nu}(x)\right)^{\prime \prime} .
$$


Since $\psi_{\nu}^{(\nu)}=1 / \psi_{\nu}$ is an eigenfunction of $V^{(\nu)}$ for the eigenvalue $E_{\nu}$, the most general eigenfunction (up to a multiplicative factor) of $V^{(\nu)}$ for the same eigenvalue is

$$
\Psi_{\nu}^{(\nu)}\left(x ; \lambda_{1}\right)=\psi_{\nu}^{(\nu)}(x)\left(\lambda_{1}+\int_{x_{0}}^{x} d t \frac{1}{\left(\psi_{\nu}^{(\nu)}(t)\right)^{2}}\right)=\frac{\lambda_{1}+\int_{x_{0}}^{x} d t \psi_{\nu}^{2}(t)}{\psi_{\nu}(x)}, \quad \lambda_{1} \in \mathbb{R},
$$

and the corresponding RS function is

$$
W_{\nu}^{(\nu)}\left(x ; \lambda_{1}\right)=-\left[\log \left(\frac{\lambda_{1}+\int_{x_{0}}^{x} d t \psi_{\nu}^{2}(t)}{\psi_{\nu}(x)}\right)\right]^{\prime}=-w_{\nu}(x)-\frac{\psi_{\nu}^{2}(x)}{\lambda_{1}+\int_{x_{0}}^{x} d t \psi_{\nu}^{2}(t)} .
$$

We can now use this general solution as seed function for the second DBT. Then applying $A\left(W_{\nu}^{(\nu)}\right)$ to $V^{(\nu)}$, we obtain the following second extension

$$
\begin{aligned}
\widetilde{V}^{(\nu, \nu)}\left(x ; \lambda_{1}\right) & =V^{(\nu)}(x)-2\left(\log \Psi_{\nu}^{(\nu)}\left(x ; \lambda_{1}\right)\right)^{\prime \prime}=V(x)-2\left[\log \left(\psi_{\nu}(x) \Psi_{\nu}^{(\nu)}\left(x ; \lambda_{1}\right)\right)\right]^{\prime \prime} \\
& =V(x)-2\left[\log \left(\lambda_{1}+\int_{x_{0}}^{x} d t \psi_{\nu}^{2}(t)\right)\right]^{\prime \prime}=V(x)-2\left(\frac{\psi_{\nu}^{2}(x)}{\lambda_{1}+\int_{x_{0}}^{x} d t \psi_{\nu}^{2}(t)}\right)^{\prime},
\end{aligned}
$$

and we recover the first Fernández formula (3.2) with $\lambda_{1}=-W_{0}$. As for the eigenfunctions of $\widetilde{V}^{(\nu, \nu)}$, they are given by $(\mu \neq \nu)$

$$
\widetilde{\psi}_{\mu}^{(\nu, \nu)}\left(x ; \lambda_{1}\right)=\widehat{A}\left(W_{\nu}^{(\nu)}\right) \psi_{\mu}^{(\nu)}(x)=\left(E_{\nu}-E_{\mu}\right) \psi_{\mu}(x)-\frac{W\left(\psi_{\nu}, \psi_{\mu} \mid x\right)}{\Psi_{\nu}^{(\nu)}\left(x ; \lambda_{1}\right)} .
$$

\subsection{General multi-step confluent chains}

Fernández and Salinas-Hernández [21] have also considered the so-called "hyperconfluent" case corresponding to a three-step confluent DBT, for which they have extended the previous formulas, equations (3.2) and (3.3). For these three-step extensions, the potential depends on two arbitrary real parameters.

In fact, the preceding analysis allows to obtain integral formulas "à la Fernández" for chains of arbitrary order in a very simple way. In the following, the symbol $\left(\nu^{l}\right)$ means $\underbrace{(\nu, \ldots, \nu)}_{l \text { times }}$.

In the three-step case, the image of $\Psi_{\nu}^{(\nu)}$ (see equation (3.5)) by the DBT $A\left(W_{\nu}^{(\nu)}\right.$ ) being $\psi_{\nu}^{\left(\nu^{2}\right)}=1 / \Psi_{\nu}^{(\nu)}$, the general eigenfunction of $V^{\left(\nu^{2}\right)}\left(x ; \lambda_{1}\right)$ (see equation (3.6)) associated to the eigenvalue $E_{\nu}$ is

$$
\Psi_{\nu}^{\left(\nu^{2}\right)}\left(x ; \Lambda_{2}\right)=\frac{\lambda_{2}+\int_{x_{0}}^{x} d t\left(\Psi_{\nu}^{(\nu)}\left(t ; \lambda_{1}\right)\right)^{2}}{\Psi_{\nu}^{(\nu)}\left(x ; \lambda_{1}\right)}, \quad \lambda_{1}, \lambda_{2} \in \mathbb{R}
$$

where we have used the notation $\Lambda_{m}=\left(\lambda_{1}, \ldots, \lambda_{m}\right)$. The next extension generated by the DBT $A\left(W_{\nu}^{\left(\nu^{2}\right)}\right)$ is then

$$
\begin{aligned}
\widetilde{V}^{\left(\nu^{3}\right)}\left(x ; \Lambda_{2}\right) & =V(x)-2\left[\log \left(\psi_{\nu}(x) \Psi_{\nu}^{(\nu)}\left(x ; \lambda_{1}\right) \Psi_{\nu}^{\left(\nu^{2}\right)}\left(x ; \Lambda_{2}\right)\right)\right]^{\prime \prime} \\
& =V(x)-2\left(\log \psi_{\nu}(x)\right)^{\prime \prime}-2\left(\lambda_{2}+\int_{x_{0}}^{x} d t\left(\Psi_{\nu}^{(\nu)}\left(t ; \lambda_{1}\right)\right)^{2}\right)^{\prime \prime} .
\end{aligned}
$$

We recover the "hyperconfluent" third-order superpartner of $V(x)$ as obtained by Fernández and Salinas-Hernández [21]. Within this scheme, the generalization is immediate and repeating the procedure $m$ times, we obtain for the hyperconfluent $m^{\text {th }}$-order extension of $V(x)$ the 
expression

$$
\widetilde{V}^{\left(\nu^{m}\right)}\left(x ; \Lambda_{m-1}\right)=V(x)-2\left(\log \prod_{j=0}^{m-1} \Psi_{\nu}^{\left(\nu^{j}\right)}\left(x ; \Lambda_{j}\right)\right)^{\prime \prime}=V(x)-2 \sum_{j=0}^{m-1}\left(\log \Psi_{\nu}^{\left(\nu^{j}\right)}\left(x ; \Lambda_{j}\right)\right)^{\prime \prime},
$$

with the following recurrence relation for the successive seed functions

$$
\Psi_{\nu}^{\left(\nu^{k}\right)}\left(x ; \Lambda_{k}\right)=\frac{\lambda_{k}+\int_{x_{0}}^{x} d t\left(\Psi_{\nu}^{\left(\nu^{k-1}\right)}\left(t ; \Lambda_{k-1}\right)\right)^{2}}{\Psi_{\nu}^{\left(\nu^{k-1}\right)}\left(x ; \Lambda_{k-1}\right)}
$$

where $\Psi_{\nu}^{(0)}\left(x ; \Lambda_{0}\right)=\psi_{\nu}(x)$.

In other words, whenever $m$ is even $(m=2 k)$

$$
\widetilde{V}^{\left(\nu^{2 k}\right)}\left(x ; \Lambda_{2 k-1}\right)=V(x)-2\left\{\log \left[\prod_{l=0}^{k-1}\left(\lambda_{2 l+1}+\int_{x_{0}}^{x} d t\left(\Psi_{\nu}^{\left(\nu^{2 l}\right)}\left(t ; \Lambda_{2 l}\right)\right)^{2}\right)\right]\right\}^{\prime \prime}
$$

and whenever $m$ is odd $(m=2 k+1)$

$$
\widetilde{V}^{\left(\nu^{2 k+1}\right)}\left(x ; \Lambda_{2 k}\right)=V(x)-2\left\{\log \left[\psi_{\nu}(x) \prod_{l=1}^{k}\left(\lambda_{2 l}+\int_{x_{0}}^{x} d t\left(\Psi_{\nu}^{\left(\nu^{2 l-1}\right)}\left(t ; \Lambda_{2 l-1}\right)\right)^{2}\right)\right]\right\}^{\prime \prime} .
$$

The eigenstates of $\widetilde{V}^{\left(\nu^{m}\right)}$ can be obtained by successive applications of the $\widehat{A}\left(W_{\nu}^{\left(\nu^{l}\right)}\right)$ operators (the product being ordered in decreasing order),

$$
\widetilde{\psi}_{k}^{\left(\nu^{m}\right)}\left(x ; \Lambda_{m-1}\right)=\prod_{l=1}^{m} \widehat{A}\left(W_{\nu}^{\left(\nu^{l-1}\right)}\right) \psi_{k}(x) .
$$

A direct application of the Crum Wronskian formulas [14] to these general, parameterdependent, confluent extensions is obviously not possible and, as mentioned above, the Matveev formulas $[27,34,35]$ correspond only to a particular choice of the $\lambda_{j}$ parameters. Nevertheless, these extended potentials are amenable to other (standard) Wronskian formulas [7, 20, 48].

In the following, we limit our analysis to the case of two-step DBT. We are interested in the possibility of building regular and rational extensions with such confluent chains, which turns out to be possible for the trigonometric Darboux-Pöschl-Teller (TDPT) potential and the isotonic potential.

\section{Two-step confluent rational extensions of the trigonometric Darboux-Pöschl-Teller (TDPT) potential}

\subsection{General scheme}

The trigonometric Darboux-Pöschl-Teller (TDPT) potential (with zero ground-state energy) is defined on $x \in] 0, \pi / 2[$ by

$$
V(x ; \alpha, \beta)=\frac{(\alpha+1 / 2)(\alpha-1 / 2)}{\sin ^{2} x}+\frac{(\beta+1 / 2)(\beta-1 / 2)}{\cos ^{2} x}-(\alpha+\beta+1)^{2},
$$

with $\alpha, \beta>1 / 2$. 
Its physical spectrum, associated to the asymptotic Dirichlet boundary conditions

$$
\psi\left(0^{+} ; \alpha, \beta\right)=0=\psi\left(\left(\frac{\pi}{2}\right)^{-} ; \alpha, \beta\right),
$$

is given in terms of Jacobi polynomials $[18,29,50]$

$$
P_{n}^{(\alpha, \beta)}(z)=\frac{(-1)^{n} \Gamma(n+\beta+1)}{n ! \Gamma(n+\alpha+\beta+1)} \sum_{k=0}^{n}(-1)^{k}\left(\begin{array}{l}
n \\
k
\end{array}\right) \frac{\Gamma(n+\alpha+\beta+1+k)}{2^{k} \Gamma(\beta+1+k)}(1+z)^{k},
$$

by

$$
\begin{aligned}
& E_{n}(\alpha, \beta)=\left(\alpha_{n}+\beta_{n}+1\right)^{2}-(\alpha+\beta+1)^{2}=4 n(\alpha+\beta+1+n), \\
& \psi_{n}(x ; \alpha, \beta)=\psi_{0}(x ; \alpha, \beta) P_{n}^{(\alpha, \beta)}(z), \quad n \in \mathbb{N},
\end{aligned}
$$

with $z=\cos 2 x \in]-1,1\left[,\left(\alpha_{n}, \beta_{n}\right)=(\alpha+n, \beta+n)\right.$, and

$$
\psi_{0}(x ; \alpha, \beta)=(1-z)^{(\alpha+1 / 2) / 2}(1+z)^{(\beta+1 / 2) / 2} .
$$

In the following, in order to get rational extensions, we consider the case where $\alpha$ and $\beta$ are integers: $\alpha=N \geq 1, \beta=M \geq 1$.

If we choose as initial seed function an eigenstate $\psi_{n}(x ; N, M)$ of $V(x ; N, M)$, by taking $x_{0}=\pi / 2\left(z_{0}=-1\right)$, the quantity

$$
Q_{n}^{(N, M)}(z)=\int_{\pi / 2}^{x} d \xi \psi_{n}^{2}(\xi ; N, M)=-\frac{1}{2} \int_{-1}^{z} d \zeta(1-\zeta)^{N}(1+\zeta)^{M}\left(P_{n}^{(N, M)}(\zeta)\right)^{2}
$$

is a polynomial of degree $N+M+2 n+1$ in $z$ with $[18,50]$

$$
\begin{aligned}
Q_{n}^{(N, M)}(1) & =-\frac{1}{2} \int_{-1}^{1} d \zeta(1-\zeta)^{N}(1+\zeta)^{M}\left(P_{n}^{(N, M)}(\zeta)\right)^{2} \\
& =-\frac{2^{N+M}}{2 n+N+M+1} \frac{(n+N) !(n+M) !}{n !(n+N+M) !}
\end{aligned}
$$

Note the following recurrence

$$
Q_{n-1}^{(N+1, M+1)}(1)=\frac{4 n}{n+N+M+1} Q_{n}^{(N, M)}(1) .
$$

From equation (3.6), we then obtain for the confluent two-step extension $\widetilde{V}^{\left(n^{2}\right)}$,

$$
\begin{aligned}
& \widetilde{V}^{\left(n^{2}\right)}\left(x ; N, M, \lambda_{1}\right)=V(x ; N, M)-2\left[\log \left(\lambda_{1}+Q_{n}^{(N, M)}(z)\right)\right]^{\prime \prime} \\
& =V(x ; N, M)+4\left(1-z^{2}\right)^{1 / 2} \frac{d}{d z}\left(\frac{(1-z)^{N+1 / 2}(1+z)^{M+1 / 2}\left(P_{n}^{(N, M)}(z)\right)^{2}}{\lambda_{1}+Q_{n}^{(N, M)}(z)}\right),
\end{aligned}
$$

where $\widetilde{V}^{\left(n^{2}\right)}\left(x ; N, M, \lambda_{1}\right)$ constitutes a rational extension of $V(x)$ (in the $z$ variable). $Q_{n}^{(N, M)}(z)$ is strictly decreasing on the interval ] $-1,1[$ and keeps a negative value, varying from 0 to $Q_{n}^{(N, M)}(1)<0$ when $z$ runs through $]-1,1[$. Consequently, when

$$
\left.\left.\left.\lambda_{1} \in\right]-\infty, 0\right] \cup\right]-Q_{n}^{(N, M)}(1),+\infty[,
$$

then $\lambda_{1}+Q_{n}^{(N, M)}(z)$ keeps a constant sign, strictly negative or strictly positive respectively, and $\widetilde{V}^{\left(n^{2}\right)}\left(x ; N, M, \lambda_{1}\right)$ is regular. 
In this case, its eigenfunctions for $k \neq n$ are given by (see equations (3.5) and (3.7))

$$
\widetilde{\psi}_{k}^{\left(n^{2}\right)}\left(x ; N, M, \lambda_{1}\right)=\left[E_{n}(N, M)-E_{k}(N, M)\right] \psi_{k}(x)-\frac{W\left(\psi_{n}, \psi_{k} \mid x\right)}{\Psi_{n}^{(n)}\left(x ; N, M, \lambda_{1}\right)},
$$

with

$$
\Psi_{n}^{(n)}\left(x ; N, M, \lambda_{1}\right)=(1-z)^{-(N+1 / 2) / 2}(1+z)^{-(M+1 / 2) / 2} \frac{\lambda_{1}+Q_{n}^{(N, M)}(z)}{P_{n}^{(N, M)}(z)}
$$

and

$$
W\left(\psi_{n}, \psi_{k} \mid x\right)=-(1-z)^{N+1}(1+z)^{M+1} P_{n, k}^{(N, M)}(z),
$$

where $\left(P_{-1}^{(\alpha, \beta)}(z)=0\right)$

$$
\begin{aligned}
P_{n, k}^{(N, M)}(z)= & (k+N+M+1) P_{n}^{(N, M)}(z) P_{k-1}^{(N+1, M+1)}(z) \\
& -(n+N+M+1) P_{n-1}^{(N+1, M+1)}(z) P_{k}^{(N, M)}(z)
\end{aligned}
$$

is an exceptional Jacobi polynomial in the broad sense of the term.

Hence, for $k \neq n$,

$$
\begin{aligned}
\widetilde{\psi}_{k}^{\left(n^{2}\right)}\left(x ; N, M, \lambda_{1}\right)= & {\left[E_{n}(N, M)-E_{k}(N, M)\right] \psi_{k}(x ; N, M) } \\
& +(1-z)^{(3 N+5 / 2) / 2}(1+z)^{(3 M+5 / 2) / 2} \frac{P_{n, k}^{(N, M)}(z) P_{n}^{(N, M)}(z)}{\lambda_{1}+Q_{n}^{(N, M)}(z)},
\end{aligned}
$$

that is

$$
\widetilde{\psi}_{k}^{\left(n^{2}\right)}\left(x ; N, M, \lambda_{1}\right)=(1-z)^{(N+1 / 2) / 2}(1+z)^{(M+1 / 2) / 2} \frac{\widetilde{P}_{N, M, k}^{\left(n^{2}\right)}\left(z ; \lambda_{1}\right)}{\lambda_{1}+Q_{n}^{(N, M)}(z)},
$$

where

$$
\begin{aligned}
\widetilde{P}_{N, M, k}^{\left(n^{2}\right)}\left(z ; \lambda_{1}\right)= & 4(n-k)(n+k+N+M+1) P_{k}^{(N, M)}(z)\left[\lambda_{1}+Q_{n}^{(N, M)}(z)\right] \\
& +(1-z)^{N+1}(1+z)^{M+1} P_{n, k}^{(N, M)}(z) P_{n}^{(N, M)}(z) .
\end{aligned}
$$

Moreover

$$
\begin{aligned}
\widetilde{\psi}_{n}^{\left(n^{2}\right)}\left(x ; N, M, \lambda_{1}\right) & =1 / \Psi_{n}^{(n)}\left(x ; N, M, \lambda_{1}\right) \\
& =(1-z)^{(N+1 / 2) / 2}(1+z)^{(M+1 / 2) / 2} \frac{P_{n}^{(N, M)}(z)}{\lambda_{1}+Q_{n}^{(N, M)}(z)},
\end{aligned}
$$

which is a normalizable eigenstate of $\widetilde{V}^{\left(n^{2}\right)}\left(x ; N, M, \lambda_{1}\right)$. This corresponds to defining

$$
\widetilde{P}_{N, M, n}^{\left(n^{2}\right)}\left(z ; \lambda_{1}\right)=P_{n}^{(N, M)}(z) .
$$

$\widetilde{\psi}_{k}^{\left(n^{2}\right)}\left(x ; N, M, \lambda_{1}\right)$ tends to zero at $z=-1$ and $z=1$ (i.e., $x=\pi / 2$ and $\left.x=0\right)$ and is then an admissible eigenstate of $\widetilde{V}^{\left(n^{2}\right)}\left(x ; N, M, \lambda_{1}\right)$ for every $k \geq 0$. The potentials $V(x ; N, M)$ and $\widetilde{V}^{\left(n^{2}\right)}\left(x ; N, M, \lambda_{1}\right)$ are therefore strictly isospectral. 
The orthogonality conditions between eigenstates imply that the $\widetilde{P}_{N, M, k}^{\left(n^{2}\right)}\left(z ; \lambda_{1}\right)$ constitute a family of orthogonal polynomials (indexed by $k \in \mathbb{N}$ ) on ]-1,1[ with respect to the measure

$$
\mu_{N, M}^{\left(n^{2}\right)}\left(z ; \lambda_{1}\right)=\frac{1}{2} \frac{(1-z)^{N}(1+z)^{M}}{\left(\lambda_{1}+Q_{n}^{(N, M)}(z)\right)^{2}} .
$$

It is worth observing here that the confluent two-step extension $\widetilde{V}^{\left(n^{2}\right)}\left(x ; N, M, \lambda_{1}\right)$ may be considered as a special case of one of those with general parameters that have been built by Contreras-Astorga and Fernández in [12, Section 3.2.3(b)] (namely the third one given in equation (3.43)), whenever their parameters $\lambda, \nu$ assume the half-integer values $\lambda=N+1 / 2$ and $\nu=M+1 / 2$.

\subsection{Examples $n=0$}

We consider the $n=0$ case. Then

$$
Q_{0}^{(N, M)}(z)=-\frac{1}{2} \int_{-1}^{z} d \zeta(1-\zeta)^{N}(1+\zeta)^{M}=-(z+1)^{M+1} \sum_{k=0}^{N} \frac{2^{N-k-1}(-1)^{k}}{M+k+1}\left(\begin{array}{c}
N \\
k
\end{array}\right)(z+1)^{k}
$$

and

$$
\begin{aligned}
\widetilde{V}^{\left(0^{2}\right)}\left(x ; N, M, \lambda_{1}\right)= & V(x ; N, M)+2 \frac{(1-z)^{2 N+1}(1+z)^{2 M+1}}{\left(\lambda_{1}+Q_{0}^{(N, M)}(z)\right)^{2}} \\
& +4(1-z)^{N}(1+z)^{M} \frac{M-N-(N+M+1) z}{\lambda_{1}+Q_{0}^{(N, M)}(z)} .
\end{aligned}
$$

Moreover

$$
\widetilde{\psi}_{k}^{\left(0^{2}\right)}\left(x ; N, M, \lambda_{1}\right)=(1-z)^{(N+1 / 2) / 2}(1+z)^{(M+1 / 2) / 2} \frac{\widetilde{P}_{N, M, k}^{\left(0^{2}\right)}\left(z ; \lambda_{1}\right)}{\lambda_{1}+Q_{0}^{(N, M)}(z)},
$$

where

$$
\begin{aligned}
\widetilde{P}_{N, M, k}^{\left(0^{2}\right)}\left(z ; \lambda_{1}\right)= & -4 k(k+N+M+1) P_{k}^{(N, M)}(z)\left(\lambda_{1}+Q_{0}^{(N, M)}(z)\right) \\
& +(1-z)^{N+1}(1+z)^{M+1} P_{0, k}^{(N, M)}(z), \quad k \neq 0, \\
\widetilde{P}_{N, M, 0}^{\left(0^{2}\right)}\left(z ; \lambda_{1}\right)= & 1,
\end{aligned}
$$

with $\left(P_{-1}^{(N, M)}(z)=0\right)$

$$
P_{0, k}^{(N, M)}(z)=(k+N+M+1) P_{k-1}^{(N+1, M+1)}(z) .
$$

\subsubsection{The $N=M=1$ case}

The results read

$$
\begin{aligned}
& Q_{0}^{(1,1)}(z)=-\frac{1}{2}(z+1)^{2}\left(1-\frac{1}{3}(z+1)\right), \\
& \widetilde{V}^{\left(0^{2}\right)}\left(x ; 1,1, \lambda_{1}\right)=V(x ; 1,1)-12 \frac{z\left(1-z^{2}\right)}{\lambda_{1}-\frac{1}{2}(z+1)^{2}+\frac{1}{6}(z+1)^{3}}
\end{aligned}
$$




$$
+2 \frac{\left(1-z^{2}\right)^{3}}{\left[\lambda_{1}-\frac{1}{2}(z+1)^{2}+\frac{1}{6}(z+1)^{3}\right]^{2}}
$$

or

$$
\begin{aligned}
\widetilde{V}^{\left(0^{2}\right)}\left(x ; 1,1, \lambda_{1}\right)= & \frac{3}{4 \sin ^{2} x}+\frac{3}{4 \cos ^{2} x}-9-12 \frac{\sin ^{2} 2 x \cos 2 x}{\lambda_{1}-2 \cos ^{4} x+\frac{4}{3} \cos ^{6} x} \\
& +2 \frac{\sin ^{6} 2 x}{\left(\lambda_{1}-2 \cos ^{4} x+\frac{4}{3} \cos ^{6} x\right)^{2}}
\end{aligned}
$$

with $\left.\left.\left.\lambda_{1} \in\right]-\infty, 0\right] \cup\right] \frac{2}{3},+\infty[$, and

$$
\widetilde{\psi}_{k}^{\left(0^{2}\right)}\left(x ; 1,1, \lambda_{1}\right)=(1-z)^{3 / 4}(1+z)^{3 / 4} \frac{\widetilde{P}_{1,1, k}^{\left(0^{2}\right)}\left(z ; \lambda_{1}\right)}{\lambda_{1}-\frac{1}{2}(z+1)^{2}+\frac{1}{6}(z+1)^{3}},
$$

where

$$
\begin{aligned}
\widetilde{P}_{1,1, k}^{\left(0^{2}\right)}\left(z ; \lambda_{1}\right)= & -4 k(k+3) P_{k}^{(1,1)}(z)\left[\lambda_{1}-\frac{1}{2}(z+1)^{2}+\frac{1}{6}(z+1)^{3}\right] \\
& +(k+3)(1-z)^{2}(1+z)^{2} P_{k-1}^{(2,2)}(z), \quad k \neq 0, \\
\widetilde{P}_{1,1,0}^{\left(0^{2}\right)}\left(z ; \lambda_{1}\right)= & 1 .
\end{aligned}
$$

\subsubsection{The $N=2, M=1$ case}

The results read

$$
\begin{aligned}
& Q_{0}^{(2,1)}(z)=-(z+1)^{2}\left(1-\frac{2}{3}(z+1)+\frac{1}{8}(z+1)^{2}\right) \\
& \widetilde{V}^{\left(0^{2}\right)}\left(x ; 2,1, \lambda_{1}\right)= \\
& \quad V(x ; 2,1)-4 \frac{(1+4 z)(1-z)^{2}(1+z)}{\lambda_{1}-(z+1)^{2}+\frac{2}{3}(z+1)^{3}-\frac{1}{8}(z+1)^{4}} \\
& +2 \frac{(1-z)^{5}(1+z)^{3}}{\left[\lambda_{1}-(z+1)^{2}+\frac{2}{3}(z+1)^{3}-\frac{1}{8}(z+1)^{4}\right]^{2}}
\end{aligned}
$$

or

$$
\begin{aligned}
\widetilde{V}^{\left(0^{2}\right)}\left(x ; 2,1, \lambda_{1}\right)= & \frac{15}{4 \sin ^{2} x}+\frac{3}{4 \cos ^{2} x}-16-32 \frac{(1-4 \cos 2 x) \sin ^{4} x \cos ^{2} x}{\lambda_{1}-4 \cos ^{4} x+\frac{16}{3} \cos ^{6} x-2 \cos ^{8} x} \\
& +512 \frac{\sin ^{10} x \cos ^{6} x}{\left(\lambda_{1}-4 \cos ^{4} x+\frac{16}{3} \cos ^{6} x-2 \cos ^{8} x\right)^{2}}
\end{aligned}
$$

with $\left.\left.\left.\lambda_{1} \in\right]-\infty, 0\right] \cup\right] \frac{2}{3},+\infty[$, and

$$
\widetilde{\psi}_{k}^{\left(0^{2}\right)}\left(x ; 2,1, \lambda_{1}\right)=(1-z)^{5 / 4}(1+z)^{3 / 4} \frac{\widetilde{P}_{2,1, k}^{\left(0^{2}\right)}\left(z ; \lambda_{1}\right)}{\lambda_{1}-(z+1)^{2}+\frac{2}{3}(z+1)^{3}-\frac{1}{8}(z+1)^{4}},
$$

where

$$
\begin{aligned}
\widetilde{P}_{2,1, k}^{\left(0^{2}\right)}\left(z ; \lambda_{1}\right)= & -4 k(k+4) P_{k}^{(2,1)}(z)\left(\lambda_{1}-(z+1)^{2}+\frac{2}{3}(z+1)^{3}-\frac{1}{8}(z+1)^{4}\right) \\
& +(k+4)(1-z)^{3}(1+z)^{2} P_{k-1}^{(3,2)}(z), \quad k \neq 0, \\
\widetilde{P}_{2,1,0}^{\left(0^{2}\right)}\left(z ; \lambda_{1}\right)= & 1 .
\end{aligned}
$$




\subsubsection{The $N=1, M=2$ case}

The results read

$$
\begin{aligned}
& Q_{0}^{(1,2)}(z)=-(z+1)^{3}\left(\frac{1}{3}-\frac{1}{8}(z+1)\right) \\
& \widetilde{V}^{\left(0^{2}\right)}\left(x ; 1,2, \lambda_{1}\right)=V(x ; 1,2)+4 \frac{(1-4 z)(1-z)(1+z)^{2}}{\lambda_{1}-\frac{1}{3}(z+1)^{3}+\frac{1}{8}(z+1)^{4}} \\
& +2 \frac{(1-z)^{3}(1+z)^{5}}{\left[\lambda_{1}-\frac{1}{3}(z+1)^{3}+\frac{1}{8}(z+1)^{4}\right]^{2}}
\end{aligned}
$$

or

$$
\begin{aligned}
\widetilde{V}^{\left(0^{2}\right)}\left(x ; 1,2, \lambda_{1}\right)= & \frac{3}{4 \sin ^{2} x}+\frac{15}{4 \cos ^{2} x}-16+32 \frac{(1-4 \cos 2 x) \sin ^{2} x \cos ^{4} x}{\lambda_{1}-\frac{8}{3} \cos ^{6} x+2 \cos ^{8} x} \\
& +512 \frac{\sin ^{6} x \cos ^{10} x}{\left(\lambda_{1}-\frac{8}{3} \cos ^{6} x+2 \cos ^{8} x\right)^{2}}
\end{aligned}
$$

with $\left.\left.\left.\lambda_{1} \in\right]-\infty, 0\right] \cup\right] \frac{2}{3},+\infty[$, and

$$
\widetilde{\psi}_{k}^{\left(0^{2}\right)}\left(x ; 1,2, \lambda_{1}\right)=(1-z)^{3 / 4}(1+z)^{5 / 4} \frac{\widetilde{P}_{1,2, k}^{\left(0^{2}\right)}\left(z ; \lambda_{1}\right)}{\lambda_{1}-\frac{1}{3}(z+1)^{3}+\frac{1}{8}(z+1)^{4}},
$$

where

$$
\begin{aligned}
\widetilde{P}_{1,2, k}^{\left(0^{2}\right)}\left(z ; \lambda_{1}\right)= & -4 k(k+4) P_{k}^{(1,2)}(z)\left(\lambda_{1}-\frac{1}{3}(z+1)^{3}+\frac{1}{8}(z+1)^{4}\right) \\
& +(k+4)(1-z)^{2}(1+z)^{3} P_{k-1}^{(2,3)}(z), \quad k \neq 0, \\
\widetilde{P}_{1,2,0}^{\left(0^{2}\right)}\left(z ; \lambda_{1}\right)= & 1 .
\end{aligned}
$$

\subsection{Shape invariance of the two-step confluent rational extensions of the TDPT potential}

$V(x ; N, M)$ is a translationally shape invariant potential with a SUSY partner

$$
V_{\mathrm{SUSY}}(x ; N, M)=V^{(0)}(x ; N, M)=V(x ; N+1, M+1)+E_{1}(N, M)
$$

and (the coefficient is readily established by a direct calculation)

$$
\psi_{n}^{(0)}(x ; N, M)=\frac{W\left(\psi_{0}, \psi_{n} \mid x\right)}{\psi_{0}(x ; N, M)}=\left(-\frac{E_{n}(N, M)}{4 n}\right) \psi_{n-1}(x ; N+1, M+1) .
$$

Since $\widetilde{V}^{\left(n^{2}\right)}\left(x ; N, M, \lambda_{1}\right)$ and $V(x ; N, M)$ are strictly isospectral, it is natural to wonder whether $\widetilde{V}^{\left(n^{2}\right)}\left(x ; N, M, \lambda_{1}\right)$ shares the same invariance property as $V(x ; N, M)$.

Consider the SUSY partner of $\widetilde{V}^{\left(n^{2}\right)}\left(x ; N, M, \lambda_{1}\right)$. It is given by

$$
\begin{aligned}
& \widetilde{V}_{\mathrm{SUSY}}^{\left(n^{2}\right)}\left(x ; N, M, \lambda_{1}\right)=\widetilde{V}^{\left(n^{2}\right)}\left(x ; N, M, \lambda_{1}\right)-2\left(\log \widetilde{\psi}_{0}^{\left(n^{2}\right)}\left(x ; N, M, \lambda_{1}\right)\right)^{\prime \prime} \\
& \quad=V(x ; N, M)-2\left[\log \left(\psi_{n}(x ; N, M) \Psi_{n}^{(n)}\left(x ; N, M, \lambda_{1}\right) \widetilde{\psi}_{0}^{\left(n^{2}\right)}\left(x ; N, M, \lambda_{1}\right)\right)\right]^{\prime \prime},
\end{aligned}
$$

where, for $n \geq 1$,

$$
\widetilde{\psi}_{0}^{\left(n^{2}\right)}\left(x ; N, M, \lambda_{1}\right)=E_{n}(N, M) \psi_{0}(x ; N, M)-\frac{W\left(\psi_{n}, \psi_{0} \mid x\right)}{\Psi_{n}^{(n)}\left(x ; N, M, \lambda_{1}\right)}
$$


and, for $n=0$,

$$
\widetilde{\psi}_{0}^{\left(0^{2}\right)}\left(x ; N, M, \lambda_{1}\right)=\frac{1}{\Psi_{0}^{(0)}\left(x ; N, M, \lambda_{1}\right)} .
$$

Consequently, in the $n=0$ case, we have

$$
\begin{aligned}
\widetilde{V}_{\mathrm{SUSY}}^{\left(0^{2}\right)}\left(x ; N, M, \lambda_{1}\right)= & V(x ; N, M) \\
& -2\left[\log \left(\psi_{0}(x ; N, M) \Psi_{0}^{(0)}\left(x ; N, M, \lambda_{1}\right) \widetilde{\psi}_{0}^{\left(0^{2}\right)}\left(x ; N, M, \lambda_{1}\right)\right)\right]^{\prime \prime} \\
& =V(x ; N, M)-2\left[\log \psi_{0}(x ; N, M)\right]^{\prime \prime},
\end{aligned}
$$

that is

$$
\widetilde{V}_{\mathrm{SUSY}}^{\left(0^{2}\right)}\left(x ; N, M, \lambda_{1}\right)=V^{(0)}(x ; N, M)=V(x ; N+1, M+1)+E_{1}(N, M) .
$$

Furthermore, in the $n \geq 1$ case, we get

$$
\begin{aligned}
\widetilde{V}_{\mathrm{SUSY}}^{\left(n^{2}\right)}( & \left.; N, M, \lambda_{1}\right)=V(x ; N, M)-2\left[\log \psi_{0}(x ; N, M)\right]^{\prime \prime} \\
& -2\left\{\log \left[\left(E_{n}(N, M) \Psi_{n}^{(n)}\left(x ; N, M, \lambda_{1}\right)+\frac{W\left(\psi_{0}, \psi_{n} \mid x\right)}{\psi_{0}(x ; N, M)}\right) \psi_{n}(x ; N, M)\right]\right\}^{\prime \prime} \\
= & V(x ; N+1, M+1)+E_{1}(N, M) \\
& -2\left\{\log \left[\left(E_{n}(N, M) \Psi_{n}^{(n)}\left(x ; N, M, \lambda_{1}\right)-\frac{E_{n}(N, M)}{4 n} \psi_{n-1}(x ; N+1, M+1)\right)\right]\right. \\
& \left.+\log \left[\psi_{n}(x ; N, M)\right]\right\}^{\prime \prime} .
\end{aligned}
$$

More precisely

$$
\begin{aligned}
\widetilde{V}_{\mathrm{SUSY}}^{\left(n^{2}\right)}( & \left.x ; N, M, \lambda_{1}\right)=V(x ; N+1, M+1)-2\left[\log \psi_{n-1}(x ; N+1, M+1)\right]^{\prime \prime}+E_{1}(N, M) \\
& -2\left(\log \frac{\lambda_{1}+Q_{n}^{(N, M)}(z)-\psi_{n}(x ; N, M) \psi_{n-1}(x ; N+1, M+1) / 4 n}{\psi_{n-1}(x ; N+1, M+1)}\right)^{\prime \prime} \\
= & V^{(n-1)}(x ; N+1, M+1)+E_{1}(N, M) \\
& -2\left(\log \frac{\lambda_{1}+Q_{n}^{(N, M)}(z)-\psi_{n}(x ; N, M) \psi_{n-1}(x ; N+1, M+1) / 4 n}{\psi_{n-1}(x ; N+1, M+1)}\right)^{\prime \prime} .
\end{aligned}
$$

If, for an appropriate constant $C$, the following condition

$$
\lambda_{1}+Q_{n}^{(N, M)}(z)-\frac{\psi_{n}(x ; N, M) \psi_{n-1}(x ; N+1, M+1)}{4 n}=C\left(\lambda_{1}^{\prime}+Q_{n-1}^{(N+1, M+1)}(z)\right)
$$

is satisfied, then

$$
\frac{\lambda_{1}+Q_{n}^{(N, M)}(z)-\psi_{n}(x ; N, M) \psi_{n-1}(x ; N+1, M+1) / 4 n}{\psi_{n-1}(x ; N+1, M+1)}=C \Psi_{n-1}^{(n-1)}\left(x ; N+1, M+1, \lambda_{1}^{\prime}\right)
$$

and we obtain an enlarged shape invariance property

$$
\widetilde{V}_{\text {SUSY }}^{\left(n^{2}\right)}\left(x ; N, M, \lambda_{1}\right)=\widetilde{V}^{\left((n-1)^{2}\right)}\left(x ; N+1, M+1, \lambda_{1}^{\prime}\right)+E_{1}(N, M) .
$$

The preceding condition (4.4) can be rewritten as

$$
\left.A(z)-B(z)=C \lambda_{1}^{\prime}-\lambda_{1}, \quad \forall z \in\right]-1,1[
$$


with

$$
\begin{aligned}
A(z)= & Q_{n}^{(N, M)}(z)-C Q_{n-1}^{(N+1, M+1)}(z) \\
= & \frac{1}{2} \int_{-1}^{z} d \zeta\left[C(1-\zeta)^{N+1}(1+\zeta)^{M+1}\left(P_{n-1}^{(N+1, M+1)}(\zeta)\right)^{2}\right. \\
& \left.-(1-\zeta)^{N}(1+\zeta)^{M}\left(P_{n}^{(N, M)}(\zeta)\right)^{2}\right]
\end{aligned}
$$

and

$$
\begin{aligned}
B(z) & =\psi_{n}(x ; N, M) \psi_{n-1}(x ; N+1, M+1) / 4 n \\
& =\frac{(1-z)^{N+1}(1+z)^{M+1}}{4 n} P_{n}^{(N, M)}(z) P_{n-1}^{(N+1, M+1)}(z) .
\end{aligned}
$$

Equation (4.6) is equivalent to

$$
\frac{d}{d z} A(z)=\frac{d}{d z} B(z)
$$

where $\frac{d}{d z} A(z)$ is given by

$$
\frac{2}{(1-z)^{N}(1+z)^{M}} \frac{d}{d z} A(z)=C\left(1-z^{2}\right)\left(P_{n-1}^{(N+1, M+1)}(z)\right)^{2}-\left(P_{n}^{(N, M)}(z)\right)^{2} .
$$

As for $\frac{d}{d z} B(z)$, using the derivation formula $[18,50]$

$$
\frac{d}{d z} P_{n}^{(N, M)}(z)=\frac{N+M+n+1}{2} P_{n-1}^{(N+1, M+1)}(z),
$$

it can be expressed as

$$
\begin{gathered}
\frac{4 n}{(1-z)^{N}(1+z)^{M}} \frac{d}{d z} B(z)=\left(1-z^{2}\right) P_{n}^{(N, M)}(z) \frac{d}{d z} P_{n-1}^{(N+1, M+1)}(z) \\
+\left(1-z^{2}\right) \frac{N+M+n+1}{2}\left(P_{n-1}^{(N+1, M+1)}(z)\right)^{2} \\
+[(M-N)-z(N+M+2)] P_{n}^{(N, M)}(z) P_{n-1}^{(N+1, M+1)}(z),
\end{gathered}
$$

that is

$$
\begin{aligned}
& \frac{4 n}{(1-z)^{N}(1+z)^{M}} \frac{d}{d z} B(z)=\left(1-z^{2}\right) \frac{N+M+n+1}{2}\left(P_{n-1}^{(N+1, M+1)}(z)\right)^{2} \\
& +\left(1-z^{2}\right) P_{n}^{(N, M)}(z) \frac{d}{d z} P_{n-1}^{(N+1, M+1)}(z) \\
& +[(M-N)-z(N+M+2)] P_{n}^{(N, M)}(z) P_{n-1}^{(N+1, M+1)}(z) .
\end{aligned}
$$

But the differential equation satisfied by the Jacobi polynomials is $[18,50]$

$$
\begin{gathered}
\left(1-z^{2}\right) \frac{d^{2}}{d z^{2}} P_{n}^{(N, M)}(z)+[(M-N)-z(N+M+2)] \frac{d}{d z} P_{n}^{(N, M)}(z) \\
=-n(N+M+n+1) P_{n}^{(N, M)}(z),
\end{gathered}
$$

which, combined with equation (4.8), gives

$$
\left(1-z^{2}\right) \frac{d}{d z} P_{n-1}^{(N+1, M+1)}(z)+[(M-N)-z(N+M+2)] P_{n-1}^{(N+1, M+1)}(z)=-2 n P_{n}^{(N, M)}(z) .
$$


Consequently

$$
\begin{aligned}
& \frac{4 n}{(1-z)^{N}(1+z)^{M}} \frac{d}{d z} B(z) \\
& \quad=-2 n\left(P_{n}^{(N, M)}(z)\right)^{2}+\left(1-z^{2}\right) \frac{N+M+n+1}{2}\left(P_{n-1}^{(N+1, M+1)}(z)\right)^{2},
\end{aligned}
$$

or

$$
\begin{aligned}
& \frac{2}{(1-z)^{N}(1+z)^{M}} \frac{d}{d z} B(z) \\
& \quad=\left(1-z^{2}\right) \frac{N+M+n+1}{4 n}\left(P_{n-1}^{(N+1, M+1)}(z)\right)^{2}-\left(P_{n}^{(N, M)}(z)\right)^{2} .
\end{aligned}
$$

To satisfy the equality (4.7), we then must choose

$$
C=\frac{N+M+n+1}{4 n} .
$$

In this case, equation (4.6) simply becomes

$$
A(z)-B(z)=\frac{N+M+n+1}{4 n} \lambda_{1}^{\prime}-\lambda_{1} .
$$

In the limit $z \rightarrow 1^{-}$, we obviously get

$$
B(1)=0,
$$

and (see equations (4.1) and (4.2))

$$
A(1)=Q_{n}^{(N, M)}(1)-\frac{N+M+n+1}{4 n} Q_{n-1}^{(N+1, M+1)}(1)=0,
$$

which implies

$$
\lambda_{1}^{\prime}=\frac{4 n}{N+M+n+1} \lambda_{1} .
$$

We conclude that for $n \geq 1$, the two-step confluent rational extensions of the TDPT potential satisfy the enlarged shape invariance property (4.5) with $\lambda_{1}^{\prime}$ given in equation (4.9). Note that the latter relation ensures that the domain of $\lambda_{1}$ values for which $\widetilde{V}^{\left(n^{2}\right)}\left(x ; N, M, \lambda_{1}\right)$ is regular corresponds exactly to the domain of $\lambda_{1}^{\prime}$ values for which $\widetilde{V}^{\left((n-1)^{2}\right)}\left(x ; N+1, M+1, \lambda_{1}^{\prime}\right)$ is also regular (see equations (4.2) and (4.3)).

In the $n=N=M=1$ case, such a property can be directly verified as follows. We get

$$
P_{0}^{(2,2)}(z)=1, \quad P_{1}^{(1,1)}(z)=2 z,
$$

$C=1, \lambda_{1}^{\prime}=\lambda_{1}$, as well as

$$
\begin{aligned}
A(z) & =Q_{1}^{(1,1)}(z)-Q_{0}^{(2,2)}(z)=\frac{1}{2} \int_{-1}^{z} d \zeta\left(\left(1-\zeta^{2}\right)^{2}-4 \zeta^{2}\left(1-\zeta^{2}\right)\right) \\
& =\frac{1}{2} \int_{-1}^{z} d \zeta\left(1-6 \zeta^{2}+5 \zeta^{4}\right)=\frac{1}{2} z\left(1-z^{2}\right)^{2}
\end{aligned}
$$

and

$$
B(z)=\frac{\left(1-z^{2}\right)^{2}}{4} 2 z .
$$

Hence the identity

$$
A(z)=B(z), \quad \forall z \in]-1,1[,
$$

is satisfied, which implies that

$$
\widetilde{V}_{\mathrm{SUSY}}^{\left(1^{2}\right)}\left(x ; 1,1, \lambda_{1}\right)=\widetilde{V}^{\left((0)^{2}\right)}\left(x ; 2,2, \lambda_{1}\right)+E_{1}(1,1) .
$$




\section{Two-step confluent rational extensions of the isotonic potential}

\subsection{General scheme}

The isotonic oscillator potential (with zero ground-state energy $E_{0}=0$ ) is defined on the positive half-line $] 0,+\infty[$ by

$$
V(x ; \omega, \alpha)=\frac{\omega^{2}}{4} x^{2}+\frac{(\alpha+1 / 2)(\alpha-1 / 2)}{x^{2}}-\omega(\alpha+1), \quad|\alpha|>1 / 2 .
$$

If we add Dirichlet boundary conditions at zero and infinity and if we suppose $\alpha>1 / 2$, it has the following spectrum $\left(z=\omega x^{2} / 2\right)$

$$
E_{n}(\omega)=2 n \omega, \quad \psi_{n}(x ; \omega, \alpha)=z^{(\alpha+1 / 2) / 2} e^{-z / 2} L_{n}^{\alpha}(z), \quad n \geq 0,
$$

where

$$
L_{n}^{\alpha}(z)=\sum_{k=0}^{n} \frac{(-1)^{k}}{k !} \frac{(n+\alpha) \cdots(k+1+\alpha)}{(n-k) !} z^{k}
$$

is the usual Laguerre polynomial $[18,50]$.

In the following, in order to get rational extensions, we consider the case where $\alpha$ is an integer: $\alpha=N \geq 1$.

If we choose as initial seed function an (unnormalized) eigenstate $\psi_{n}(x ; \omega, N)$ of $V(x ; \omega, N)$, by taking $x_{0}=0$ we get

$$
\begin{aligned}
\int_{0}^{x} d t \psi_{n}^{2}(t ; \omega, N) & =\left\|\psi_{n}\right\|^{2}-\int_{x}^{+\infty} d t \psi_{n}^{2}(t ; \omega, N) \\
& =\left\|\psi_{n}\right\|^{2}-\frac{1}{\sqrt{2 \omega}} \int_{z}^{+\infty} d \zeta \zeta^{N}\left(L_{n}^{N}(\zeta)\right)^{2} \exp (-\zeta) .
\end{aligned}
$$

But

$$
F_{n}^{N}(z)=-\frac{1}{\sqrt{2 \omega}} \int_{z}^{+\infty} d \zeta \zeta^{N}\left(L_{n}^{N}(\zeta)\right)^{2} \exp (-\zeta)=\frac{1}{\sqrt{2 \omega}} \exp (-z) Q_{n}^{N}(z),
$$

where

$$
Q_{n}^{N}(z)=-\sum_{j=0}^{N+2 n} \frac{d^{j}}{d z^{j}}\left(z^{N}\left(L_{n}^{N}(z)\right)^{2}\right)
$$

is a polynomial of degree $N+2 n$.

In contrast with what happened in the TDPT case, to obtain a rational extension we need now to eliminate the exponential factor, which necessitates fixing $\lambda_{1}=-\left\|\psi_{n}\right\|^{2}$. Equation (3.6) then becomes

$$
\begin{aligned}
\widetilde{V}^{\left(n^{2}\right)}\left(x ; \omega, N,-\left\|\psi_{n}\right\|^{2}\right) & =V(x ; \omega, N)-2\left[\log \left(F_{n}^{N}(z)\right)\right]^{\prime \prime} \\
& =V(x ; \omega, N)-4 \omega z^{1 / 2} \frac{d}{d z}\left(\frac{z^{N+1 / 2}\left(L_{n}^{N}(z)\right)^{2}}{Q_{n}^{N}(z)}\right),
\end{aligned}
$$

where $\widetilde{V}^{\left(n^{2}\right)}\left(x ; \omega, N,-\left\|\psi_{n}\right\|^{2}\right)$ constitutes a rational extension of $V(x ; \omega, N)$. Since $F_{n}^{N}(z)$ is a negative, strictly increasing function of $z$, such that

$$
\lim _{x \rightarrow+\infty} F_{n}^{N}(z)=0
$$


$Q_{n}^{N}(z)$ keeps a constant strictly negative sign, i.e., $Q_{n}^{N}(z)$ has no zero on the positive half-line. This shows that $\widetilde{V}^{\left(n^{2}\right)}\left(x ; \omega, N,-\left\|\psi_{n}\right\|^{2}\right)$ is also regular.

Let us note too that $[18,50]$

$$
F_{n}^{N}(0)=-\frac{1}{\sqrt{2 \omega}} \int_{0}^{+\infty} d \zeta \zeta^{N}\left(L_{n}^{N}(\zeta)\right)^{2} \exp (-\zeta)=-\frac{1}{\sqrt{2 \omega}} \frac{(n+N) !}{n !}<0,
$$

or

$$
Q_{n}^{N}(0)=-\frac{(n+N) !}{n !} .
$$

The eigenfunctions of $\widetilde{V}^{\left(n^{2}\right)}\left(x ; \omega, N,-\left\|\psi_{n}\right\|^{2}\right)$ are given by (see equation (3.7))

$$
\widetilde{\psi}_{k}^{\left(n^{2}\right)}\left(x ; \omega, N,-\left\|\psi_{n}\right\|^{2}\right)=\left[E_{n}(\omega)-E_{k}(\omega)\right] \psi_{k}(x ; \omega, N)-\frac{W\left(\psi_{n}, \psi_{k} \mid x\right)}{\Psi_{n}^{(n)}\left(x ; \omega, N,-\left\|\psi_{n}\right\|^{2}\right)}, \quad k \neq n,
$$

where

$$
\begin{aligned}
\Psi_{n}^{(n)}\left(x ; \omega, N,-\left\|\psi_{n}\right\|^{2}\right) & =\frac{\int_{0}^{x} d t \psi_{n}^{2}(t ; \omega, N)-\left\|\psi_{n}\right\|^{2}}{\psi_{n}(x ; \omega, N)} \\
& =\frac{F_{n}^{N}(z)}{\psi_{n}(x ; \omega, N)}=\frac{1}{\sqrt{2 \omega}} z^{-(N+1 / 2) / 2} \exp (-z / 2) \frac{Q_{n}^{N}(z)}{L_{n}^{N}(z)}
\end{aligned}
$$

and

$$
W\left(\psi_{n}, \psi_{k} \mid x\right)=\sqrt{2 \omega} z^{N+1} \exp (-z) L_{n, k}^{N}(z)
$$

with

$$
L_{n, k}^{N}(z)=L_{n-1}^{N+1}(z) L_{k}^{N}(z)-L_{n}^{N}(z) L_{k-1}^{N+1}(z) .
$$

Consequently

$$
\begin{aligned}
\widetilde{\psi}_{k}^{\left(n^{2}\right)}\left(x ; \omega, N,-\left\|\psi_{n}\right\|^{2}\right)= & {\left[E_{n}(\omega)-E_{k}(\omega)\right] \psi_{k}(x ; \omega, N) } \\
& -2 \omega z^{(3 N+5 / 2) / 2} \exp (-z / 2) \frac{L_{n, k}^{N}(z) L_{n}^{N}(z)}{Q_{n}^{N}(z)},
\end{aligned}
$$

that is,

$$
\widetilde{\psi}_{k}^{\left(n^{2}\right)}\left(x ; \omega, N,-\left\|\psi_{n}\right\|^{2}\right)=\frac{2 \omega z^{(N+1 / 2) / 2} \exp (-z / 2)}{Q_{n}^{N}(z)} \widetilde{L}_{k, N}^{\left(n^{2}\right)}(z),
$$

where

$$
\widetilde{L}_{k, N}^{\left(n^{2}\right)}(z)=(n-k) L_{k}^{N}(z) Q_{n}^{N}(z)-z^{N+1} L_{n, k}^{N}(z) L_{n}^{N}(z)
$$

$\widetilde{\psi}_{k}^{\left(n^{2}\right)}\left(x ; \omega, N,-\left\|\psi_{n}\right\|^{2}\right)$ decreases exponentially to zero at infinity and also tends to zero at the origin (see equation (5.5)). It is therefore an admissible eigenstate of $\widetilde{V}^{\left(n^{2}\right)}\left(x ; \omega, N,-\left\|\psi_{n}\right\|^{2}\right)$ for every $k \neq n$.

Note that in this case

$$
\widetilde{\psi}_{n}^{\left(n^{2}\right)}\left(x ; \omega, N,-\left\|\psi_{n}\right\|^{2}\right)=1 / \Psi_{n}^{(n)}\left(x ; \omega, N,-\left\|\psi_{n}\right\|^{2}\right) \propto z^{(N+1 / 2) / 2} \exp (z / 2) \frac{L_{n}^{N}(z)}{Q_{n}^{N}(z)}
$$


is not an eigenstate since it is not normalizable. This implies that $\widetilde{V}^{\left(n^{2}\right)}\left(x ; \omega, N,-\left\|\psi_{n}\right\|^{2}\right)$ and $V(x ; \omega, N)$ are only quasi-isospectral, the two-step confluent DBT $A\left(W_{\nu}^{(\nu)}\right) \circ A\left(w_{\nu}\right)$ being now state-deleting.

The orthogonality conditions between eigenstates imply that the set of $\widetilde{L}_{k, N}^{\left(n^{2}\right)}(z)$ with $k \neq n$, constitute a family of orthogonal polynomials, indexed by $k \in \mathbb{N}$, on the positive half-line with respect to the mesure

$$
\mu_{N}^{\left(n^{2}\right)}(z)=\frac{z^{N} \exp (-z)}{\left(Q_{n}^{N}(z)\right)^{2}}
$$

\subsection{Examples}

\subsubsection{The $n=0$ case}

The $n=0$ case gives no new extended potentials with respect to previously known results. Indeed, the corresponding confluent two-step DBT is obtained by applying successively the onestep DBT $A\left(w_{0}\right)$ and $A\left(W_{0}^{(0)}\right)$. The first one is the usual SUSY partnership and, due to the shape invariance property of the isotonic potential, we have

$$
V^{(0)}(x ; \omega, N)=V(x ; \omega, N+1)+2 \omega,
$$

where $V^{(0)}(x ; \omega, N)$ admits the following eigenstates

$$
\psi_{k}^{(0)}(x ; \omega, N) \sim \psi_{k-1}(x ; w, N+1),
$$

for the respective energies $E_{k}(\omega), k \geq 1$.

The second DBT $A\left(W_{0}^{(0)}\right)$ is associated to the seed function $\Psi_{0}^{(0)}\left(x ; \omega, N,-\left\|\psi_{0}\right\|^{2}\right)$, which is a formal eigenfunction of $V^{(0)}(x ; \omega, N)$ for the eigenvalue $E_{0}=0$ and which is then in the disconjugacy sector of $V^{(0)}(x ; \omega, N)$. But

$$
\Psi_{0}^{(0)}\left(x ; \omega, N, \lambda_{1}\right)=\psi_{0}^{(0)}(x ; \omega, N)\left(\lambda_{1}+\int_{x_{0}}^{x} d t \frac{1}{\left(\psi_{0}^{(0)}(t ; \omega, N)\right)^{2}}\right),
$$

where

$$
\psi_{0}^{(0)}(x ; \omega, N) \sim 1 / \psi_{0}(x ; \omega, N)=z^{-(N+1 / 2) / 2} e^{+z / 2} .
$$

By taking $x_{0}=0$ and $\lambda_{1}=-\left\|\psi_{0}\right\|^{2}$, we obtain

$$
\Psi_{0}^{(0)}\left(x ; \omega, N,-\left\|\psi_{0}\right\|^{2}\right)=\frac{1}{\sqrt{2 \omega}} z^{-(N+1 / 2) / 2} \exp (-z / 2) Q_{0}^{N}(z),
$$

where

$$
Q_{0}^{N}(z)=-\sum_{j=0}^{N} \frac{d^{j}}{d z^{j}}\left(z^{N}\right)=-N ! \sum_{l=0}^{N} \frac{z^{l}}{l !}
$$

which gives

$$
\Psi_{0}^{(0)}\left(x ; \omega, N,-\left\|\psi_{0}\right\|^{2}\right)=-\frac{N !}{\sqrt{2 \omega}} \exp (-z / 2) z^{-(N+1 / 2) / 2} \sum_{l=0}^{N} \frac{z^{l}}{l !} .
$$


Equation (5.9) shows that $\Psi_{0}^{(0)}\left(x ; \omega, N,-\left\|\psi_{0}\right\|^{2}\right)$ is a formal eigenfunction of $V^{(0)}(x ; \omega, N)=$ $V(x ; \omega, N+1)+2 \omega$ that tends to zero at infinity and diverges at the origin. Such an eigenfunction is unique (up to a constant multiplicative factor) at a given eigenvalue (here $E_{0}=0$ ) $[6,30]$.

Consequently $\Psi_{0}^{(0)}\left(x ; \omega, N,-\left\|\psi_{0}\right\|^{2}\right)$ coincides necessarily (up to a constant multiplicative factor) with a type II seed function [22] of $V(x ; \omega, N+1)+2 \omega$, which is a formal eigenfunction for the eigenvalue $E_{-1}+2 \omega$, namely

$$
\Psi_{0}^{(0)}\left(x ; \omega, N,-\left\|\psi_{0}\right\|^{2}\right) \sim \phi_{N,-}(x ; \omega, N+1)=z^{-(N+1 / 2) / 2} e^{-z / 2} L_{N}^{-N-1}(z),
$$

where $\phi_{n,-}$ is defined by

$$
\phi_{n,-}(x ; \omega, \alpha)=\psi_{n}(x ; \omega,-\alpha) .
$$

Note that this result can be recovered directly by using the identity (see equation (5.1))

$$
L_{N}^{-N-1}(z)=(-1)^{N} \sum_{l=0}^{N} \frac{z^{l}}{l !}
$$

in equation (5.9).

Applying then the DBT $A\left(W_{0}^{(0)}\right)=A\left(v_{N,-}(x ; \omega, N+1)\right)\left(v_{N,-}=-\phi_{N,-}^{\prime} / \phi_{N,-}\right)$ to $V^{(0)}(x ;$ $\omega, N)=V(x ; \omega, N+1)+2 \omega$, we arrive at a two-step confluent extended potential

$$
\widetilde{V}^{\left(0^{2}\right)}\left(x ; \omega, N,-\left\|\psi_{0}\right\|^{2}\right)=V^{(N,-)}(x ; \omega, N+1)+2 \omega,
$$

which coincides (up to a constant shift) with the usual extensions obtained with type II seed functions [22].

Explicitly, we get

$$
\widetilde{V}^{\left(0^{2}\right)}\left(x ; \omega, N,-\left\|\psi_{0}\right\|^{2}\right)=V(x ; \omega, N)+\frac{4 \omega}{N !} z^{1 / 2} \frac{d}{d z}\left[z^{N+1 / 2} /\left(\sum_{l=0}^{N} z^{l} / l !\right)\right]
$$

and

$$
\widetilde{\psi}_{k}^{\left(0^{2}\right)}\left(x ; \omega, N,-\left\|\psi_{0}\right\|^{2}\right) \sim \widetilde{L}_{k, N}^{\left(0^{2}\right)}(z) \frac{z^{(N+1 / 2) / 2} \exp (-z / 2)}{\sum_{l=0}^{N} z^{l} / l !}, \quad k \geq 1 .
$$

In the $N=1$ case, this gives

$$
\widetilde{V}^{\left(0^{2}\right)}\left(x ; \omega, 1,-\left\|\psi_{0}\right\|^{2}\right)=\frac{\omega^{2}}{4} x^{2}+\frac{3}{4 x^{2}}+\frac{4 \omega}{\omega x^{2}+2}-\frac{16 \omega}{\left(\omega x^{2}+2\right)^{2}}=V^{(1,-)}(x ; \omega, 2)+2 \omega
$$

and

$$
\widetilde{\psi}_{k}^{\left(0^{2}\right)}\left(x ; \omega, 1,-\left\|\psi_{0}\right\|^{2}\right) \sim \frac{2 \omega z^{3 / 4} \exp (-z / 2)}{z+1} \widetilde{L}_{k, 1}^{\left(0^{2}\right)}(z), \quad k \geq 1
$$

with

$$
\widetilde{L}_{k, 1}^{\left(0^{2}\right)}(z)=k L_{k}^{1}(z)(z+1)+z^{2} L_{k-1}^{2}(z) .
$$




\subsubsection{The $n=1$ case}

We have in this case

$$
Q_{1}^{N}(z)=-z^{N+2}+N z^{N+1}-(N+1) ! \sum_{j=0}^{N} \frac{z^{j}}{j !}
$$

and

$$
\begin{gathered}
\widetilde{V}^{\left(1^{2}\right)}\left(x ; \omega, N,-\left\|\psi_{1}\right\|^{2}\right)=V(x ; \omega, N)-4 \omega \frac{z^{N}(N+1-z)}{Q_{1}^{N}(z)}\left[\left(N+\frac{1}{2}\right)(N+1)-\left(N+\frac{5}{2}\right) z\right] \\
-4 \omega \frac{z^{N+1}(N+1-z)^{2}}{\left[Q_{1}^{N}(z)\right]^{2}}\left((N+2) z^{N+1}-N(N+1) z^{N}+(N+1) ! \sum_{j=0}^{N-1} \frac{z^{j}}{j !}\right) .
\end{gathered}
$$

The eigenstates of $\widetilde{V}^{\left(1^{2}\right)}$ are $(k \neq 1)$

$$
\widetilde{\psi}_{k}^{\left(1^{2}\right)}\left(x ; \omega, N,-\left\|\psi_{1}\right\|^{2}\right)=(1-k) \psi_{k}(x ; \omega, N)-z^{(3 N+5 / 2) / 2} \exp (-z / 2) \frac{L_{1, k}^{N}(z) L_{1}^{N}(z)}{Q_{1}^{N}(z)},
$$

that is,

$$
\widetilde{\psi}_{k}^{\left(1^{2}\right)}\left(x ; \omega, N,-\left\|\psi_{1}\right\|^{2}\right)=\frac{2 \omega z^{(N+1 / 2) / 2} \exp (-z / 2)}{Q_{1}^{N}(z)} \widetilde{L}_{k, N}^{\left(1^{2}\right)}(z)
$$

where

$$
\begin{aligned}
\widetilde{L}_{k, N}^{\left(1^{2}\right)}(z)= & z^{N+1}(N+1-z)^{2} L_{k-1}^{N+1}(z) \\
& +\left(k z^{N+2}-(N k+1) z^{N+1}+(k-1)(N+1) ! \sum_{j=0}^{N} \frac{z^{j}}{j !}\right) L_{k}^{N}(z) .
\end{aligned}
$$

In particular, for $N=1$

$$
Q_{1}^{1}(z)=-z^{3}+z^{2}-2 z-2
$$

and

$$
\widetilde{V}^{\left(1^{2}\right)}\left(x ; \omega, 1,-\left\|\psi_{1}\right\|^{2}\right)=V(x ; \omega, 1)+6 \omega \frac{z^{2}-4}{z^{3}-z^{2}+2 z+2}-20 \omega \frac{5 z^{2}-4 z-2}{\left(z^{3}-z^{2}+2 z+2\right)^{2}}+2 \omega,
$$

or

$$
\begin{aligned}
\widetilde{V}^{\left(1^{2}\right)}\left(x ; \omega, 1,-\left\|\psi_{1}\right\|^{2}\right)= & \frac{\omega^{2}}{4} x^{2}+\frac{3}{4 x^{2}}+12 \omega \frac{\omega^{2} x^{4}-16}{\omega^{3} x^{6}-2 \omega^{2} x^{4}+8 \omega x^{2}+16} \\
& -320 \omega \frac{5 \omega^{2} x^{4}-8 \omega x^{2}-8}{\left(\omega^{3} x^{6}-2 \omega^{2} x^{4}+8 \omega x^{2}+16\right)^{2}} .
\end{aligned}
$$

Moreover $(k \neq 1)$

$$
\widetilde{\psi}_{k}^{\left(1^{2}\right)}\left(x ; \omega, 1,-\left\|\psi_{1}\right\|^{2}\right)=-\frac{2 \omega z^{3 / 4} \exp (-z / 2)}{z^{3}-z^{2}+2 z+2} \widetilde{L}_{k, 1}^{\left(1^{2}\right)}(z)
$$

where

$$
\widetilde{L}_{k, 1}^{\left(1^{2}\right)}(z)=\left[k z^{3}-(k+1) z^{2}+2(k-1) z+2(k-1)\right] L_{k}^{1}(z)+z^{2}(2-z)^{2} L_{k-1}^{2}(z) .
$$


The polynomial

$$
\widetilde{L}_{0,1}^{\left(1^{2}\right)}(z)=-\left(z^{2}+2 z+2\right)
$$

is associated to the ground state of $\widetilde{V}^{\left(1^{2}\right)}\left(x ; \omega, 1,-\left\|\psi_{1}\right\|^{2}\right)$ and

$$
\widetilde{L}_{2,1}^{\left(1^{2}\right)}(z)=-\frac{1}{2}\left(z^{4}+4 z^{2}-12\right),
$$

which has a single zero at $\sqrt{2}$ on $] 0,+\infty[$, corresponds to the first excited eigenstate.

\subsection{Shape invariance of the two-step confluent rational extensions of the isotonic potential}

$V(x ; \omega, N)$ is a translationally shape invariant potential with a SUSY partner

$$
V_{\mathrm{SUSY}}(x ; \omega, N)=V^{(0)}(x ; \omega, N)=V(x ; \omega, N+1)+E_{1}(\omega)
$$

and (the coefficient is readily established by a direct calculation)

$$
\psi_{n}^{(0)}(x ; \omega, N)=\frac{W\left(\psi_{0}, \psi_{n} \mid x\right)}{\psi_{0}(x ; \omega, N)}=-\sqrt{2 \omega} \psi_{n-1}(x ; \omega, N+1) .
$$

Considering the SUSY partner of $\widetilde{V}^{\left(n^{2}\right)}\left(x ; \omega, N,-\left\|\psi_{n}\right\|^{2}\right)$, let us deal separately with the $n \geq 1$ and $n=0$ cases. For $n \geq 1$, this partner is given by

$$
\begin{aligned}
& \widetilde{V}_{\mathrm{SUSY}}^{\left(n^{2}\right)}\left(x ; \omega, N,-\left\|\psi_{n}\right\|^{2}\right)=\widetilde{V}^{\left(n^{2}\right)}\left(x ; \omega, N,-\left\|\psi_{n}\right\|^{2}\right)-2\left(\log \widetilde{\psi}_{0}^{\left(n^{2}\right)}\left(x ; \omega, N,-\left\|\psi_{n}\right\|^{2}\right)\right)^{\prime \prime}(5.11) \\
& \quad=V(x ; \omega, N)-2\left[\log \left(\psi_{n}(x ; \omega, N) \Psi_{n}^{(n)}\left(x ; \omega, N,-\left\|\psi_{n}\right\|^{2}\right) \widetilde{\psi}_{0}^{\left(n^{2}\right)}\left(x ; \omega, N,-\left\|\psi_{n}\right\|^{2}\right)\right)\right]^{\prime \prime},
\end{aligned}
$$

where

$$
\widetilde{\psi}_{0}^{\left(n^{2}\right)}\left(x ; \omega, N,-\left\|\psi_{n}\right\|^{2}\right)=E_{n}(\omega) \psi_{0}(x ; \omega, N)-\frac{W\left(\psi_{n}, \psi_{0} \mid x\right)}{\Psi_{n}^{(n)}\left(x ; \omega, N,-\left\|\psi_{n}\right\|^{2}\right)} .
$$

Inserting (5.12) into (5.11), we get

$$
\begin{aligned}
\widetilde{V}_{\mathrm{SUSY}}^{\left(n^{2}\right)}( & \left.x ; \omega, N,-\left\|\psi_{n}\right\|^{2}\right)=V(x ; \omega, N)-2\left(\log \psi_{0}(x ; \omega, N)\right)^{\prime \prime} \\
& -2\left\{\log \left[\left(E_{n}(\omega) \Psi_{n}^{(n)}\left(x ; \omega, N,-\left\|\psi_{n}\right\|^{2}\right)+\frac{W\left(\psi_{0}, \psi_{n} \mid x\right)}{\psi_{0}(x ; \omega, N)}\right) \psi_{n}(x ; \omega, N)\right]\right\}^{\prime \prime} \\
= & V(x ; \omega, N+1)+E_{1}(\omega) \\
& -2\left\{\log \left[\left(2 n \omega \Psi_{n}^{(n)}\left(x ; \omega, N,-\left\|\psi_{n}\right\|^{2}\right)-\sqrt{2 \omega} \psi_{n-1}(x ; \omega, N+1)\right) \psi_{n}(x ; \omega, N)\right]\right\}^{\prime \prime} .
\end{aligned}
$$

More precisely, on using (5.6),

$$
\begin{aligned}
\widetilde{V}_{\mathrm{SUSY}}^{\left(n^{2}\right)}( & \left.x ; \omega, N,-\left\|\psi_{n}\right\|^{2}\right)=V(x ; \omega, N+1)-2\left(\log \psi_{n-1}(x ; \omega, N+1)\right)^{\prime \prime}+E_{1}(\omega) \\
& -2\left\{\log \left[\left(F_{n}^{N}(z)-\frac{\psi_{n}(x ; \omega, N) \psi_{n-1}(x ; \omega, N+1)}{\sqrt{2 \omega} n}\right) / \psi_{n-1}(x ; \omega, N+1)\right]\right\}^{\prime \prime} \\
= & V^{(n-1)}(x ; \omega, N+1)+E_{1}(\omega) \\
& -2\left\{\log \left[\left(F_{n}^{N}(z)-\frac{\psi_{n}(x ; \omega, N) \psi_{n-1}(x ; \omega, N+1)}{\sqrt{2 \omega} n}\right) / \psi_{n-1}(x ; \omega, N+1)\right]\right\}^{\prime \prime} .
\end{aligned}
$$


If, for an appropriate constant $C$, the following condition

$$
F_{n}^{N}(z)-\frac{\psi_{n}(x ; \omega, N) \psi_{n-1}(x ; \omega, N+1)}{\sqrt{2 \omega} n}=C F_{n-1}^{N+1}(z)
$$

is satisfied, then

$$
\begin{gathered}
\left(F_{n}^{N}(z)-\frac{\psi_{n}(x ; \omega, N) \psi_{n-1}(x ; \omega, N+1)}{\sqrt{2 \omega} n}\right) / \psi_{n-1}(x ; \omega, N+1) \\
=C \Psi_{n-1}^{(n-1)}\left(x ; \omega, N+1,-\left\|\psi_{n-1}\right\|^{2}\right)
\end{gathered}
$$

and we obtain an enlarged shape invariance property

$$
\widetilde{V}_{\mathrm{SUSY}}^{\left(n^{2}\right)}\left(x ; \omega, N,-\left\|\psi_{n}\right\|^{2}\right)=\widetilde{V}^{\left((n-1)^{2}\right)}\left(x ; \omega, N+1,-\left\|\psi_{n-1}\right\|^{2}\right)+E_{1}(\omega) .
$$

The preceding condition (5.13) can be rewritten as

$$
A(z)-B(z)=0, \quad \forall z \in]-1,1[,
$$

with

$$
A(z)=F_{n}^{N}(z)-C F_{n-1}^{N+1}(z)=\frac{1}{\sqrt{2 \omega}} \int_{z}^{+\infty} d \zeta\left(C \zeta^{N+1}\left(L_{n-1}^{N+1}(\zeta)\right)^{2}-\zeta^{N}\left(L_{n}^{N}(\zeta)\right)^{2}\right) \exp (-\zeta)
$$

and

$$
B(z)=\frac{\psi_{n}(x ; \omega, N) \psi_{n-1}(x ; \omega, N+1)}{\sqrt{2 \omega} n}=\frac{z^{N+1} e^{-z} L_{n}^{N}(z) L_{n-1}^{N+1}(z)}{\sqrt{2 \omega} n} .
$$

Equation (5.15) is equivalent to the set of conditions

$$
\frac{d}{d z} A(z)=\frac{d}{d z} B(z)
$$

and

$$
A(0)=B(0) .
$$

Here $\frac{d}{d z} A(z)$ is given by

$$
\sqrt{2 \omega} \frac{d}{d z} A(z)=\left(\left(L_{n}^{N}(z)\right)^{2}-C z\left(L_{n-1}^{N+1}(z)\right)^{2}\right) z^{N} \exp (-z) .
$$

As for $\frac{d}{d z} B(z)$, using the derivation formula $[18,50]$

$$
\frac{d}{d z} L_{n}^{N}(z)=-L_{n-1}^{N+1}(z)
$$

it can be expressed as

$$
\begin{aligned}
\sqrt{2 \omega} n \frac{d}{d z} B(z)= & -z^{N} \exp (-z) L_{n}^{N}(z)\left(z \frac{d^{2}}{d z^{2}} L_{n}^{N}(z)+(N+1-z) \frac{d}{d z} L_{n}^{N}(z)\right) \\
& -z^{N+1} \exp (-z)\left(L_{n-1}^{N+1}(z)\right)^{2} .
\end{aligned}
$$

The differential equation satisfied by the Laguerre polynomials is [18, 50]

$$
z \frac{d^{2}}{d z^{2}} L_{n}^{N}(z)+(N+1-z) \frac{d}{d z} L_{n}^{N}(z)=-n L_{n}^{N}(z),
$$


which, inserted in equation (5.18), yields

$$
\sqrt{2 \omega} \frac{d}{d z} B(z)=\left(\left(L_{n}^{N}(z)\right)^{2}-\frac{1}{n} z\left(L_{n-1}^{N+1}(z)\right)^{2}\right) z^{N} \exp (-z) .
$$

Hence, to satisfy the first condition (5.16), we must choose

$$
C=\frac{1}{n}
$$

With such a choice for $C$, the second condition (5.17) is then also fulfilled because it is obvious that $B(0)=0$ and, from equation (5.4), it results that

$$
A(0)=F_{n}^{N}(0)-\frac{1}{n} F_{n-1}^{N+1}(0)=0 .
$$

We conclude that for any $n \geq 1$, the two-step confluent extension $\widetilde{V}_{\mathrm{SUSY}}^{\left(n^{2}\right)}\left(x ; \omega, N,-\left\|\psi_{n}\right\|^{2}\right)$ satisfies the enlarged shape invariant property (5.14).

Such a property is in particular satisfied if $n=N=1$, as it can be directly verified. In this case, we indeed have

$$
L_{0}^{(2)}(z)=1, \quad L_{1}^{(1)}(z)=2-z,
$$

and $C=1$, so that the identity (see equations (5.2) and (5.3))

$$
\begin{aligned}
A(z) & =F_{1}^{1}(z)-F_{0}^{2}(z)=\frac{1}{\sqrt{2 \omega}} \exp (-z)\left(Q_{1}^{1}(z)-Q_{0}^{2}(z)\right) \\
& =\frac{1}{\sqrt{2 \omega}} \exp (-z)\left(\sum_{j=0}^{2} \frac{d^{j}}{d z^{j}}\left(z^{2}\right)-\sum_{j=0}^{3} \frac{d^{j}}{d z^{j}}\left(z(2-z)^{2}\right)\right)=\frac{z^{2}(2-z)}{\sqrt{2 \omega}} e^{-z}=B(z)
\end{aligned}
$$

is fulfilled, which implies that

$$
\widetilde{V}_{\mathrm{SUSY}}^{\left(1^{2}\right)}\left(x ; \omega, 1,-\left\|\psi_{1}\right\|^{2}\right)=\widetilde{V}^{\left((0)^{2}\right)}\left(x ; \omega, 2,-\left\|\psi_{0}\right\|^{2}\right)+E_{1}(\omega) .
$$

Let us now turn ourselves to the $n=0$ case. As noted above (see equation (5.7)), the formal eigenfunction

$$
\widetilde{\psi}_{0}^{\left(0^{2}\right)}\left(x ; \omega, N,-\left\|\psi_{0}\right\|^{2}\right)=\frac{1}{\Psi_{0}^{(0)}\left(x ; \omega, N,-\left\|\psi_{0}\right\|^{2}\right)},
$$

associated to the eigenvalue $E_{0}=0$, is not an eigenstate of $\widetilde{V}^{\left(0^{2}\right)}\left(x ; \omega, N,-\left\|\psi_{0}\right\|^{2}\right)$ and the ground state of this potential is actually given by $\widetilde{\psi}_{1}^{\left(0^{2}\right)}\left(x ; \omega, N,-\left\|\psi_{0}\right\|^{2}\right)$. The SUSY partner therefore reads

$$
\begin{aligned}
& \widetilde{V}_{\mathrm{SUSY}}^{\left(0^{2}\right)}\left(x ; \omega, N,-\left\|\psi_{0}\right\|^{2}\right)=\widetilde{V}^{\left(0^{2}\right)}\left(x ; \omega, N,-\left\|\psi_{0}\right\|^{2}\right)-2\left(\log \widetilde{\psi}_{1}^{\left(0^{2}\right)}\left(x ; \omega, N,-\left\|\psi_{0}\right\|^{2}\right)\right)^{\prime \prime} \\
& \quad=V(x ; \omega, N)-2\left[\log \left(\psi_{0}(x ; \omega, N) \Psi_{0}^{(0)}\left(x ; \omega, N,-\left\|\psi_{0}\right\|^{2}\right) \widetilde{\psi}_{1}^{\left(0^{2}\right)}\left(x ; \omega, N,-\left\|\psi_{0}\right\|^{2}\right)\right)\right]^{\prime \prime},
\end{aligned}
$$

where

$$
\widetilde{\psi}_{1}^{\left(0^{2}\right)}\left(x ; \omega, N,-\left\|\psi_{0}\right\|^{2}\right)=-E_{1}(\omega) \psi_{1}(x ; \omega, N)-\frac{W\left(\psi_{0}, \psi_{1} \mid x\right)}{\Psi_{0}^{(0)}\left(x ; \omega, N,-\left\|\psi_{0}\right\|^{2}\right)} .
$$


Using the shape invariance property of $V$, we can then write

$$
\begin{aligned}
& \widetilde{V}_{\mathrm{SUSY}}^{\left(0^{2}\right)}\left(x ; \omega, N,-\left\|\psi_{0}\right\|^{2}\right)=V(x ; \omega, N+1)+E_{1}(\omega) \\
& \quad-2\left[\log \left(-2 \omega \psi_{1}(x ; \omega, N) \Psi_{0}^{(0)}\left(x ; \omega, N,-\left\|\psi_{0}\right\|^{2}\right)-W\left(\psi_{0}, \psi_{1} \mid x\right)\right)\right]^{\prime \prime},
\end{aligned}
$$

that is, with equation (5.8) and (5.10),

$$
\begin{aligned}
\widetilde{V}_{\mathrm{SUSY}}^{\left(0^{2}\right)}( & \left.x ; \omega, N,-\left\|\psi_{0}\right\|^{2}\right)=V(x ; \omega, N+1)+E_{1}(\omega)-2\left[\log \psi_{0}(x ; \omega, N)\right]^{\prime \prime} \\
& -2\left[\log \left(L_{1}^{N}(z) z^{-(N+1 / 2) / 2} \exp (-z / 2) Q_{0}^{N}(z)-\psi_{0}(x ; \omega, N+1)\right)\right]^{\prime \prime} \\
= & V^{(0)}(x ; \omega, N+1)+E_{1}(\omega) \\
& -2\left\{\log \left[z^{-(N+3 / 2) / 2} \exp (-z / 2)\left(L_{1}^{N}(z) Q_{0}^{N}(z)-z^{N+1}\right)\right]\right\}^{\prime \prime} .
\end{aligned}
$$

If there existed some constant $C$ such that

$$
L_{1}^{N}(z) Q_{0}^{N}(z)-z^{N+1}=C Q_{0}^{N+1}(z)
$$

then we would get

$$
\widetilde{V}_{\mathrm{SUSY}}^{\left(0^{2}\right)}\left(x ; \omega, N,-\left\|\psi_{0}\right\|^{2}\right)=\widetilde{V}^{\left(0^{2}\right)}(x ; \omega, N+1)+E_{1}(\omega) .
$$

However, it can be readily seen that equation (5.19) cannot be satisfied by any $C$, so that we conclude that we do not have any strict nor enlarged shape invariance for the confluent extension $\widetilde{V}^{\left(0^{2}\right)}$.

\section{Final comments}

Using two-step confluent chains of DBT, we have generated new families of orthogonal polynomials, associated to novel regular rational extensions of the isotonic and TDPT potentials, exhibiting an enlarged shape invariance property. Interestingly, in the second case, the orthogonal polynomials depend on a free parameter that can be modulated continuously, a feature already encountered for other extensions based on para-Jacobi polynomials.

Considering chains of arbitrary order and the possibility of obtaining more general families of orthogonal polynomials subjected to multi-parameter dependence would be a very interesting topic for future investigation.

As a final point, it is worth observing that apart from the Darboux (or differential) approach to the construction of (quasi-)isospectral families of potentials, considered in the present paper, there also exist the Abraham-Moses [1] and Pursey [40] (or integral) approaches to the same, coming from the inverse scattering Gel'fand-Levitan or Marchenko technique. Relations between both types of methods have been extensively studied by several authors (see, e.g., [5, 33, 38, 46]), showing that they sometimes lead to the same results, but are in general inequivalent. The differential procedure being more convenient and easier to use in quantum mechanics has been preferred here. Studying the relation between our results and those of the Abraham-Moses method would, however, be an interesting open question for future work.

\section{References}

[1] Abraham P.B., Moses H.E., Changes in potentials due to changes in the point spectrum: anharmonic oscillators with exact solutions, Phys. Rev. A 22 (1980), 1333-1340. 
[2] Adler M., Moser J., On a class of polynomials connected with the Korteweg-de Vries equation, Comm. Math. Phys. 61 (1978), 1-30.

[3] Adler V.È., On a modification of Crum's method, Theoret. and Math. Phys. 101 (1994), 1381-1386.

[4] Bagchi B., Grandati Y., Quesne C., Rational extensions of the trigonometric Darboux-Pöschl-Teller potential based on para-Jacobi polynomials, J. Math. Phys. 56 (2015), 062103, 11 pages, arXiv:1411.7857.

[5] Baye D., Phase-equivalent potentials for arbitrary modifications of the bound spectrum, Phys. Rev. A 48 (1993), 2040-2047.

[6] Berezin F.A., Shubin M.A., The Schrödinger equation, Kluwer, Dordrecht, 1991.

[7] Bermudez D., Fernández C. D.J., Fernández-García N., Wronskian differential formula for confluent supersymmetric quantum mechanics, Phys. Lett. A 376 (2012), 692-696, arXiv:1109.0079.

[8] Burchnall J.L., Chaundy T.W., A set of differential equations which can be solved by polynomials, Proc. London Math. Soc. S2-30 (1930), 401-414.

[9] Calogero F., Yi G., Can the general solution of the second-order ODE characterizing Jacobi polynomials be polynomial?, J. Phys. A: Math. Theor. 45 (2012), 095206, 4 pages.

[10] Cariñena J.F., Ramos A., Integrability of the Riccati equation from a group-theoretical viewpoint, Internat. J. Modern Phys. A 14 (1999), 1935-1951, math-ph/9810005.

[11] Cariñena J.F., Ramos A., Fernández C. D.J., Group theoretical approach to the intertwined Hamiltonians, Ann. Physics 292 (2001), 42-66, math-ph/0311029.

[12] Contreras-Astorga A., Fernández C. D.J., Supersymmetric partners of the trigonometric Pöschl-Teller potentials, J. Phys. A: Math. Theor. 41 (2008), 475303, 18 pages, arXiv:0809.2760.

[13] Cooper F., Khare A., Sukhatme U., Supersymmetry in quantum mechanics, World Scientific Publishing Co., Inc., River Edge, NJ, 2001.

[14] Crum M.M., Associated Sturm-Liouville systems, Quart. J. Math. Oxford Ser. (2) 6 (1955), 121-127, physics/9908019.

[15] Darboux G., Sur une proposition relative aux équations linéaires, C. R. Acad. Sci. Paris 94 (1882), 14561459.

[16] Darboux G., Leçons sur la théorie générale des surfaces et les applications géométriques du calcul infinitésimal. II, 2nd ed., Gauthier-Villars, Paris, 1915.

[17] Durán A.J., Pérez M., Admissibility condition for exceptional Laguerre polynomials, J. Math. Anal. Appl. 424 (2015), 1042-1053, arXiv:1409.4901.

[18] Erdélyi A., Magnus W., Oberhettinger F., Tricomi F.G., Higher transcendental functions, McGraw-Hill, New York, 1953.

[19] Fernández C. D.J., Salinas-Hernández E., The confluent algorithm in second-order supersymmetric quantum mechanics, J. Phys. A: Math. Gen. 36 (2003), 2537-2543, quant-ph/0303123.

[20] Fernández C. D.J., Salinas-Hernández E., Wronskian formula for confluent second-order supersymmetric quantum mechanics, Phys. Lett. A 338 (2005), 13-18, quant-ph/0502147.

[21] Fernández C. D.J., Salinas-Hernández E., Hyperconfluent third-order supersymmetric quantum mechanics, J. Phys. A: Math. Theor. 44 (2011), 365302, 11 pages, arXiv:1105.2333.

[22] Gómez-Ullate D., Grandati Y., Milson R., Extended Krein-Adler theorem for the translationally shape invariant potentials, J. Math. Phys. 55 (2014), 043510, 30 pages, arXiv:1309.3756.

[23] Gómez-Ullate D., Kamran N., Milson R., An extended class of orthogonal polynomials defined by a SturmLiouville problem, J. Math. Anal. Appl. 359 (2009), 352-367, arXiv:0807.3939.

[24] Grandati Y., Solvable rational extensions of the isotonic oscillator, Ann. Physics 326 (2011), 2074-2090, arXiv:1101.0055.

[25] Grandati Y., Multistep DBT and regular rational extensions of the isotonic oscillator, Ann. Physics $\mathbf{3 2 7}$ (2012), 2411-2431, arXiv:1108.4503.

[26] Grandati Y., New rational extensions of solvable potentials with finite bound state spectrum, Phys. Lett. A 376 (2012), 2866-2872, arXiv:1203.4149.

[27] Grandati Y., A short proof of the Gaillard-Matveev theorem based on shape invariance arguments, Phys. Lett. A 378 (2014), 1755-1759, arXiv:1211.2392.

[28] Grandati Y., Bérard A., Rational solutions for the Riccati-Schrödinger equations associated to translationally shape invariant potentials, Ann. Physics 325 (2010), 1235-1259, arXiv:0910.4810. 
[29] Grandati Y., Bérard A., Comments on the generalized SUSY QM partnership for Darboux-Pöschl-Teller potential and exceptional Jacobi polynomials, J. Engrg. Math. 82 (2013), 161-171.

[30] Hartman P., Ordinary differential equations, John Wiley \& Sons, Inc., New York - London - Sydney, 1964.

[31] Keung W.-Y., Sukhatme U.P., Wang Q.M., Imbo T.D., Families of strictly isospectral potentials, J. Phys. A: Math. Gen. 22 (1989), L987-L992.

[32] Krein M.G., On a continual analogue of a Christoffel formula from the theory of orthogonal polynomials, Dokl. Akad. Nauk SSSR 113 (1957), 970-973.

[33] Luban M., Pursey D.L., New Schrödinger equations for old: inequivalence of the Darboux and AbrahamMoses constructions, Phys. Rev. D 33 (1986), 431-436.

[34] Matveev V.B., Generalized Wronskian formula for solutions of the KdV equations: first applications, Phys. Lett. A 166 (1992), 205-208.

[35] Matveev V.B., Positons: slowly decreasing analogues of solitons, Theoret. and Math. Phys. 131 (2002), 483-497.

[36] Messiah A., Mécanique quantique, Vol. 1, Dunod, Paris, 1959.

[37] Mielnik B., Nieto L.M., Rosas-Ortiz O., The finite difference algorithm for higher order supersymmetry, Phys. Lett. A 269 (2000), 70-78, quant-ph/0004024.

[38] Nieto M.M., Relationship between supersymmetry and the inverse method in quantum mechanics, Phys. Lett. B 145 (1984), 208-210.

[39] Odake S., Sasaki R., Infinitely many shape invariant potentials and new orthogonal polynomials, Phys. Lett. B 679 (2009), 414-417, arXiv:0906.0142.

[40] Pursey D.L., New families of isospectral Hamiltonians, Phys. Rev. D 33 (1986), 1048-1055.

[41] Quesne C., Exceptional orthogonal polynomials, exactly solvable potentials and supersymmetry, J. Phys. A: Math. Theor. 41 (2008), 392001, 6 pages, arXiv:0807.4087.

[42] Quesne C., Solvable rational potentials and exceptional orthogonal polynomials in supersymmetric quantum mechanics, SIGMA 5 (2009), 084, 24 pages, arXiv:0906.2331.

[43] Quesne C., Higher-order SUSY, exactly solvable potentials, and exceptional orthogonal polynomials, Modern Phys. Lett. A 26 (2011), 1843-1852, arXiv:1106.1990.

[44] Quesne C., Novel enlarged shape invariance property and exactly solvable rational extensions of the RosenMorse II and Eckart potentials, SIGMA 8 (2012), 080, 19 pages, arXiv:1208.6165.

[45] Quesne C., Revisiting (quasi-)exactly solvable rational extensions of the Morse potential, Internat. J. Modern Phys. A 27 (2012), 1250073, 18 pages, arXiv:1203.1811.

[46] Samsonov B.F., On the equivalence of the integral and the differential exact solution generation methods for the one-dimensional Schrödinger equation, J. Phys. A: Math. Gen. 28 (1995), 6989-6998.

[47] Samsonov B.F., New possibilities for supersymmetry breakdown in quantum mechanics and second-order irreducible Darboux transformations, Phys. Lett. A 263 (1999), 274-280, quant-ph/9904009.

[48] Schulze-Halberg A., Wronskian representation for confluent supersymmetric transformation chains of arbitrary order, Eur. Phys. J. Plus 128 (2013), 69, 17 pages.

[49] Sparenberg J.-M., Baye D., Supersymmetric transformations of real potentials on the line, J. Phys. A: Math. Gen. 28 (1995), 5079-5095.

[50] Szegö G., Orthogonal polynomials, American Mathematical Society Colloquium Publications, Vol. 23, 4th ed., Amer. Math. Soc., Providence, R.I., 1975.

[51] Vein R., Dale P., Determinants and their applications in mathematical physics, Applied Mathematical Sciences, Vol. 134, Springer-Verlag, New York, 1999. 